\title{
Normalized Analysis and Design of LCC Resonant Converters
}

\author{
Adam J. Gilbert, Christopher M. Bingham, David A. Stone, and Martin P. Foster
}

\begin{abstract}
A normalization of the LCC voltage-output resonant converter performance characteristics, in terms of the tank gain at resonance and the parallel-to-series-capacitor ratio, is presented. The resulting description is subsequently used for the derivation of a design procedure that incorporates the effects of diode losses and the finite charge/discharge time of the parallel capacitor. Unlike previously reported techniques, the resulting normalized behavior of the converter is used to identify design regions to facilitate a reduction in component electrical stresses, and the use of harmonics to transfer real power. Consideration of the use of preferred component values is also given. The underlying methodology is ultimately suitable for incorporation into a software suite for use as part of a rapid interactive design tool. Both simulation results and experimental measurements from a prototype converter are included to demonstrate the attributes of the proposed analysis and design methodologies.
\end{abstract}

Index Terms-Circuit analysis, dc-dc, resonant converters.

\section{NOMENCLATURE}

$\hat{X}$

$V_{i}$

$V_{o}$

$V_{s}$

$V_{d}$

$V_{\mathrm{pp}}$

$V_{\mathrm{Cs}}$

$V_{\mathrm{Cp}}$

$V_{\mathrm{Ls}}$

$I_{s}$

$I_{\mathrm{Ls}}$

$I_{\mathrm{tp}}$

$I_{\mathrm{Ls} \text { norm1 }}$

$I_{\text {Ls norm2 }}$

$P_{s}$

$P_{\text {tw }}$

$P_{o}$
" $\wedge$ " denotes the peak of any respective variable $X$.

DC Input voltage, $V$.

DC output voltage, $V$.

Switching voltage, $V$.

Diode voltage drop, $V$.

Output ripple voltage (peak-peak), $V$.

Series capacitor voltage, $V$.

Parallel capacitor voltage, $V$.

Series inductor voltage, $V$.

Excitation source current, $A$.

Series inductor current, $A$.

Transformer primary current, $A$.

Peak series inductor current normalized to a sinusoid at resonance.

Peak series inductor current normalized to that at full load.

Excitation source supplied power, $W$.

Power transferred through transformer primary, $W$.

Power delivered to load, $W$.

Manuscript received August 9, 2006; revised October 9, 2006. Recommended for publication by Associate Editor C. Canesin.

The authors are with the Department of Electronic and Electrical Engineering, University of Sheffield, Sheffield S1 3JD, UK (e-mail: c.bingham @ sheffield.ac. uk).

Digital Object Identifier 10.1109/TPEL.2007.909243

$R_{L}, R_{o}$
$R_{x}$
$C_{p}, C_{p}$ norm
$C_{f}, C_{f \text { norm }}$
$C_{s}$
$C_{e}$
$L_{s}, L_{s \text { norm }}$
$N$
$G_{\mathrm{tr}}, G_{\mathrm{tr} o}$
$G_{o}$

$f_{r}, f_{\text {ro }}, f_{r \max }$

$f_{m}$

$f_{s}$

$Q$

$E_{s}, E_{d}$

$A$

$F_{n}(\ldots)$

$\theta$

$M n$

$X_{\mathrm{Mn}}(t)$

$t_{\mathrm{Mn}}$

$Q_{\mathrm{Mn}}$
Load resistance, Nominal load resistance, $\Omega$. Nominal load scaling factor, $R_{L}=R_{x} R_{o}$. Parallel capacitor, $F$, normalized parallel capacitor.

Filter capacitor, $F$, normalized filter capacitor. Series capacitor, $F$.

Effective capacitance, $F$.

Series inductance, $H$, normalized series inductance.

Transformer turns ratio.

Tank gain at resonance, tank gain at resonance for nominal load.

Tank gain.

Resonant frequency, resonant frequency for nominal load, maximum resonant frequency, $\mathrm{Hz}$.

Resonant frequency to switching frequency scaling factor, $f_{s}=f_{m} f_{r}$.

Switching frequency.

Quality factor.

Resonant tank stored energy, Energy dissipated to load per cycle, J.

Ratio of parallel to series capacitor, $A=C_{p} / C_{s}$.

Function $n$.

Phase shift above resonance, degs.

Mode $n$.

Variable $X$ expressed over time period corresponding to mode $n$.

Time spent in mode $n, s$.

Charge transfer through series inductor during mode $n$.

\section{INTRODUCTION}

$\mathbf{P}$ OWER conversion based on the excitation of a resonant tank network is considered appropriate when high switching frequencies facilitate a significant reduction in the volume/mass of reactive circuit components. Through the use of zero-voltage switching (ZVS) and basic snubber circuitry, switching losses can be significantly reduced [1]-[4]. The voltage-output LCC resonant converter (Fig. 1), while having good part-load efficiency characteristics, is difficult to analyse as a result of the finite time taken to charge and discharge the parallel capacitor [5]-[9]. Fundamental Mode Approximation (FMA), the most widely used technique for analysis, assumes that the parallel capacitor waveform is a square-wave, which 


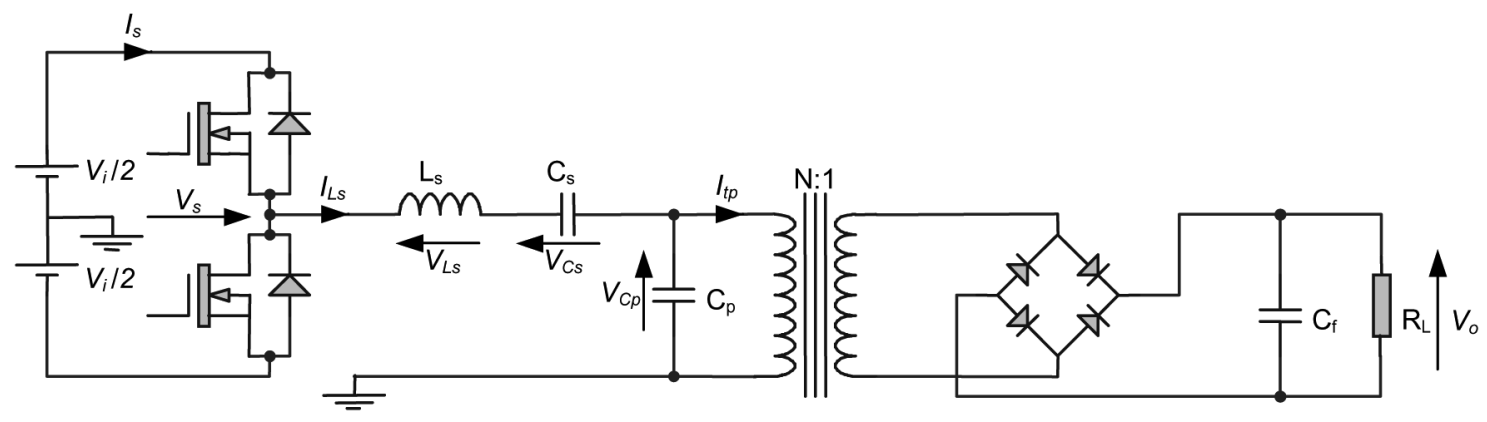

Fig. 1. LCC voltage-output resonant converter.

can significantly degrade analysis accuracy. Here, the proposed techniques inherently include the effects of the finite charge and discharge time, and normalize the converter's behavior such that the tank gain, at resonance, and the parallel- to series-capacitor ratio, fully characterize the frequency response, the resonant tank component values, the electrical stresses and the output voltage regulation performance. This is in contrast to previously published normalization techniques [10]-[12] based on the series resonant frequency, which leads to the actual system resonant frequency and gain being unknown, thereby not guaranteeing operation above the resonant frequency for a specific design. The paper therefore considers three converter operating scenarios viz. operation at the resonant frequency, and operation above resonance for low and high loading conditions. The resulting equations and graphical design curves are more readily employed than those previously reported since it is not necessary to numerically solve simultaneous equations, as required in [10], for instance. Finally, it is shown that in certain operating regions harmonics of the tank can be utilized to transfer real power which can ultimately be used as a mechanism to reduce peak electrical stresses on components. A prototype converter is used to demonstrate the benefits of the proposed design process.

The series-parallel resonant converter topology combines the advantages of the series resonant converter topology (without $C_{p}$ ) with that of the parallel resonant converter (without $C_{s}$ ). The series-resonant converter for example can only provide a gain $\left(V_{o} / V_{i}\right)<1 / 2$ when no transformer is used. The incorporation of the 'boost capacitor' $C_{p}$ allows effective input-output gains $>1 / 2$ without the necessity of a transformer [2], [13], [14].

\section{Converter Operation AT the Resonant Frequency}

When operating at the resonant frequency, four modes (M1...M4) describe the converter's behavior over a single switching period in steady-state, as defined by the example waveforms in Fig. 2. Mode M1 corresponds to the period when $C_{p}$ is charging, while M2 corresponds to the rectifier conduction period-during this period the parallel capacitor voltage $\left(V_{\mathrm{Cp}}\right)$ is clamped to the referred dc output voltage. Through symmetry, M3 and M4 are similarly defined for the second half cycle.

Converter behavior can be described by equivalent electrical networks, which, for operation during M1 and M2, are given in Fig. 3(a) and (b), respectively, which can be used to derive a piecewise description of steady-state operation.

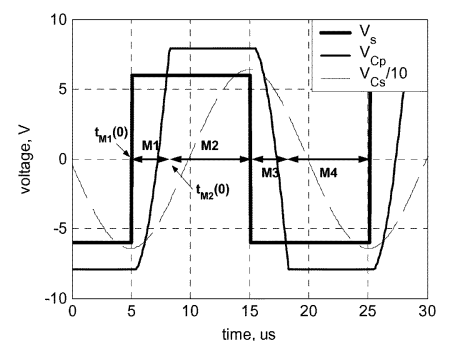

(a)

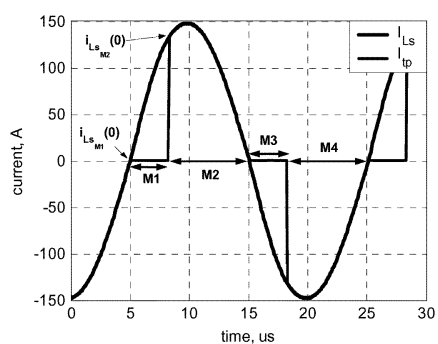

(b)
Fig. 2. Definition of modes for operation at resonance (a) voltages and (b) currents

For simplicity the charge transfer through the series inductor during the various modes will be written as follows:

$$
Q_{M n}=\int_{t_{M n}(0)}^{t_{M n}+t_{M n}(0)} i_{L s_{M n}}(t) d t
$$

where $t_{M n}$ is the total time spent in mode $n$ and $t_{M n}(0)$ is the initial time of the mode.

At resonance, the output voltage $\left(V_{o}\right)$ is given by:

$$
V_{o}=\frac{G_{\mathrm{tr}} V_{i}}{N}
$$

where $G_{\mathrm{tr}}$ is the voltage gain of the tank at resonance $\left(\hat{V}_{\mathrm{Cp}} / V_{i}\right)$. From Fig. 2(a), it can be seen that during M1 the parallel capacitor $C_{p}$ charges from the most negative clamped voltage to the most positive. The magnitude of the clamping voltage is equal to the output voltage referred to the primary winding of the transformer $\left(N V_{o}\right)$. From (2), the total charge supplied to $C_{p}$ during M1 is

$$
\begin{aligned}
\Delta V_{C p_{M 1}} & =\frac{Q_{M 1}}{C_{p}}=2 N V_{o}=2 G_{\mathrm{tr}} V_{i} \rightarrow \\
Q_{M 1} & =2 G_{\mathrm{tr}} V_{i} C_{p} .
\end{aligned}
$$

To provide the initial conditions for each mode it is instructive to consider expressions for the power flow in the converter. Assuming high efficiency operation, and the converter has sufficient filter capacitance that $V_{o}$ can be considered constant over a single switching period (consideration of rectifier losses will be addressed in Section VIII), the power provided by the excitation source, $P_{s}$, and the power entering the transformer primary 


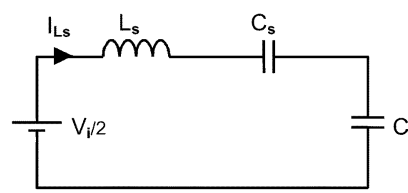

(a)

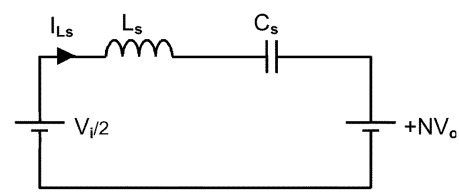

(b)
Fig. 3. Equivalent circuits for operation at the resonant frequency during (a) M1 and (b) M2.

winding, $P_{t w}$, are both approximately equal to the output power, $P_{o}$ giving

$$
\begin{aligned}
P_{s} & =V_{i} f_{r}\left(Q_{M 1}+Q_{M 2}\right) \\
P_{t w} & =2 V_{i} G_{\mathrm{tr}} f_{r} Q_{M 2}, \quad P_{s} \approx P_{t w} \approx P_{o} \\
P_{o} & =\frac{G_{\mathrm{tr}}^{2} V_{i}^{2}}{N^{2} R_{L}} .
\end{aligned}
$$

From (4), equating $P_{t w}$ and $P_{o}$ provides an expression for the total charge transferred through the primary of the transformer during a half cycle, as follows:

$$
Q_{M 2}=\frac{G_{\mathrm{tr}} V_{i}}{2 N^{2} R_{L} f_{r}} .
$$

Now, equating $P_{s}$ and $P_{t w}$ in (4), eliminating $Q_{M 1}$ and $Q_{M 2}$ using (3), (5), and solving for $C_{p}$ gives

$$
\begin{aligned}
C_{p} & =\frac{2 G_{\mathrm{tr}}-1}{4 N^{2} R_{L} f_{r}}=\frac{F_{1}\left(G_{\mathrm{tr}}\right)}{N^{2} R_{L} f_{r}} \\
\text { where } F_{1}\left(G_{\mathrm{tr}}\right) & =\frac{2 G_{\mathrm{tr}}-1}{4} .
\end{aligned}
$$

Since $C_{p}>0$, (6) provides a constraint on the minimum permissible resonant tank gain i.e., $G_{\mathrm{tr}}>1 / 2$.

The parallel- to series-capacitor ratio, $A$, is defined as

$$
A=\frac{C_{p}}{C_{s}} .
$$

For convenience, designers of LCC converters often assume $A=1$ as a good compromise between bandwidth, component sizing, electrical stresses and impedance matching. It will be shown, however, that the proposed normalization procedure can readily provide performance characteristics of the converter for alternative values of $A$, with additional benefits.

The voltage across $C_{s}$ at the start of M1 is equal to the negative of the voltage at the end of M2

$$
\begin{aligned}
V_{C s_{M 2}}\left(t_{M 2}\right) & =-V_{C s_{M 1}}(0) \\
& =V_{C s_{M 1}}(0)+\frac{1}{C_{s}}\left(Q_{M 1}+Q_{M 2}\right) .
\end{aligned}
$$

Substituting (3), (5) into (8) and solving for $V_{C s}$ at the start of M1 then gives

$$
V_{C s_{M 1}}(0)=-\frac{2 A V_{i} G_{\mathrm{tr}}^{2}}{2 G_{\mathrm{tr}}-1} .
$$

During M1, the rectifier is non-conducting and the system consists of a basic series $L C$-network, Fig. 3(a). The series and parallel capacitances effectively present a series combination, $C_{e}$, as seen from the perspective of the power devices, where

$$
C_{e}=\frac{C_{p}}{1+A} .
$$

From Fig. 3, the general solution for $i_{L s}$ during mode $n(\mathrm{M} n)$ is

$$
\begin{aligned}
& i_{L s_{M n}}(t)=i_{L s_{M n}}(0) \cos \left(\frac{t}{\sqrt{L_{s} C_{e}}}\right) \\
&+V_{L s_{M n}}(0) \sqrt{\frac{C_{e}}{L_{s}}} \sin \left(\frac{t}{\sqrt{L_{s} C_{e}}}\right)
\end{aligned}
$$

with the inductor voltage at the start of M1 being given by

$$
V_{L s_{\mathrm{M} 1}}(0)=\frac{V_{i}}{2}-V_{C s_{\mathrm{M} 1}}(0)-V_{C p_{\mathrm{M} 1}}(0) \text {. }
$$

Substituting (9) into (12) and recalling that $V_{C p_{M 1}}(0)=$ $-N V_{o}$ now gives

$$
V_{L s_{M 1}}(0)=\frac{V_{i}\left(4(A+1) G_{\mathrm{tr}}^{2}-1\right)}{2\left(2 G_{\mathrm{tr}}-1\right)} .
$$

From Fig. 2(b), it can be seen that $i_{L s_{M 1}}(0)=0$. Therefore, substituting (6), (10), and (13) into (11) gives

$$
\begin{aligned}
i_{L s_{M 1}}(t)=\frac{V_{i}\left(4(A+1) G_{\mathrm{tr}}^{2}-1\right)}{4 N \sqrt{R_{L} f_{r}(A+1) L_{s}\left(2 G_{\mathrm{tr}}-1\right)}} \\
\times \sin \left(2 N \sqrt{\frac{R_{L} f_{r}(A+1)}{L_{s}\left(2 G_{\mathrm{tr}}-1\right)}} t\right)
\end{aligned}
$$

and substituting (3), (6), and (14) into (1) and solving for $t=$ $t_{M 1}$ provides

$$
\begin{aligned}
t_{\mathrm{M} 1}=\frac{1}{2} & \sqrt{\frac{L_{s}\left(2 G_{\mathrm{tr}}-1\right)}{N^{2} R_{L} f_{r}(A+1)}} \\
& \times\left(\pi-\cos ^{-1}\left(\frac{(A+1)\left(2 G_{\mathrm{tr}}-1\right)^{2}-A}{4(A+1) G_{\mathrm{tr}}^{2}-1}\right)\right) .
\end{aligned}
$$

To determine the initial conditions for M2, (15) is substituted into (14), for $t=t_{M 1}$ giving

$$
i_{L s_{M 2}}(0)=\frac{V_{i}}{N} \sqrt{\frac{G_{\mathrm{tr}}\left(2(A+1) G_{\mathrm{tr}}-1\right)}{2 R_{L} f_{r} L_{s}}}
$$

and by differentiating (14) and inserting (15)

$$
\begin{aligned}
V_{L s_{M 2}}(0) & =L_{s} \frac{d}{d t} i_{L s_{M 1}}\left(t_{M 1}\right) \\
& =-\frac{V_{i}\left((A+1)\left(2 G_{\mathrm{tr}}-1\right)^{2}-A\right)}{2\left(2 G_{\mathrm{tr}}-1\right)} .
\end{aligned}
$$

During M2, the rectifier is conducting and $V_{C p}$ is clamped to $N V_{o}$, see Fig. 3(b), and $C_{e}$ as seen from the perspective of the power switches is given by

$$
C_{e}=\frac{C_{p}}{A} .
$$


Substituting the initial conditions (16), (17), and $C_{e}$, into (11), and eliminating $C_{p}$ using (6), leaves

$$
\begin{aligned}
i_{L s_{M 2}}(t)= & \frac{V_{i}}{N} \sqrt{\frac{G_{\mathrm{tr}}\left(2 G_{\mathrm{tr}}(A+1)-1\right)}{2 L_{s} R_{L} f_{r}}} \\
& \times \cos \left(t 2 N \sqrt{\frac{R_{L} f_{r} A}{L_{s}\left(2 G_{\mathrm{tr}}-1\right)}}\right) \\
& -\frac{V_{i}\left(G_{\mathrm{tr}} 4(A+1)\left(G_{\mathrm{tr}}-1\right)+1\right)}{4 N \sqrt{\left(2 G_{\mathrm{tr}}-1\right) L_{s} R_{L} f_{r} A}} \\
& \times \sin \left(t 2 N \sqrt{\frac{R_{L} f_{r} A}{L_{s}\left(2 G_{\mathrm{tr}}-1\right)}}\right) .
\end{aligned}
$$

Now, M2 ends when $i_{L s}=0$, hence, equating (19) to 0 , and solving for $t=t_{M 2}$ gives

$$
\begin{aligned}
t_{M 2}= & \frac{1}{2 N} \sqrt{\frac{L_{s}\left(2 G_{\mathrm{tr}}-1\right)}{A R_{L} f_{r}}} \\
& \times \cos ^{-1}\left(\frac{(1+A)\left(2 G_{\mathrm{tr}}-1\right)^{2}-A}{(1+A)\left(2 G_{\mathrm{tr}}-1\right)^{2}+2 A\left(2 G_{\mathrm{tr}}-1\right)+A}\right) .
\end{aligned}
$$

Due to symmetry, the resonant frequency is related to the mode times by

$$
f_{r}=\frac{1}{2\left(t_{M 1}+t_{M 2}\right)} .
$$

Substituting (15) and (20) into (21) gives a design value for $L_{s}$

$$
\begin{aligned}
L_{s} & =\frac{N^{2} R_{L}}{f_{r}} F_{2}\left(A, G_{\mathrm{tr}}\right) \quad \text { where } \\
F_{2}\left(A, G_{\mathrm{tr}}\right) & =\frac{1}{N^{2} R_{L} 4 f_{r}\left(\frac{t_{M 1}}{\sqrt{L_{s}}}+\frac{t_{M 2}}{\sqrt{L_{s}}}\right)^{2}}
\end{aligned}
$$

and $F_{2}$ becomes solely a function of $A$ and $G_{\mathrm{tr}}$ from the use of (15) and (20).

[Note: the $\sqrt{L_{s}}$ terms in the denominator of (22) are cancelled by numerator terms in (15) and (20) hence $F_{2}\left(A, G_{\mathrm{tr}}\right)$ does not explicitly depend on $L_{s}$ after the substitutions.]

A key factor in the operation of resonant converters is the quality factor, $Q$, at resonance, defined at the beginning of M1 as

$$
Q=2 \pi \frac{\text { Energy Stored }}{\text { Energy Dissipated/cycle }}=2 \pi \frac{E_{s}}{E_{d}} \text {. }
$$
by

The energy stored at the beginning of $\mathrm{M} 1\left(i_{\mathrm{Ls}}=0\right)$ is given

$$
E_{s}=\frac{C_{p} V_{C s_{M 1}}^{2}(0)}{2 A}+\frac{C_{p} V_{C p_{M 1}}^{2}(0)}{2}
$$

and the energy dissipated per cycle is

$$
E_{d}=\frac{V_{i}^{2} G_{\mathrm{tr}}^{2}}{N^{2} R_{L} f_{r}}
$$

TABLE I

CONVERTER SPECIFICATIONS AT RESONANCE

\begin{tabular}{|c|ccccc|}
\hline Design No. & $G_{t r}(\boldsymbol{V} / \boldsymbol{V})$ & $\boldsymbol{N}(\boldsymbol{V} / \boldsymbol{V})$ & $\boldsymbol{A}(\boldsymbol{F} / \boldsymbol{F})$ & $\boldsymbol{f}_{\boldsymbol{r}}(\mathbf{k H z})$ & $\boldsymbol{R}_{\boldsymbol{L}}(\Omega)$ \\
\hline $\mathbf{( 1 )}$ & 0.55 & 0.1 & 10 & 25 & 0.1 \\
$\mathbf{( 2 )}$ & 0.6 & 0.75 & 5 & 125 & 5 \\
$\mathbf{( 3 )}$ & 0.7 & 0.33 & 0.5 & 100 & 10 \\
$\mathbf{( 4 )}$ & 0.8 & 10 & 1 & 15 & 0.3 \\
$\mathbf{( 5 )}$ & 0.9 & 20 & 4 & 75 & 20 \\
$\mathbf{( 6 )}$ & 1 & 0.25 & 5 & 60 & 100 \\
$\mathbf{( 7 )}$ & 2 & 1 & 2 & 20 & 10 \\
$\mathbf{( 8 )}$ & 5 & 10 & 0.5 & 100 & 2 \\
$\mathbf{( 9 )}$ & 10 & 0.1 & 20 & 200 & 10 \\
$\mathbf{( 1 0 )}$ & 20 & 0.25 & 10 & 50 & 1000 \\
\hline
\end{tabular}

TABLE II

SPICE Simulation Results When Using Ideal Component Values

\begin{tabular}{|c|ccc|cc|cc|}
\cline { 2 - 8 } \multicolumn{1}{c|}{} & \multicolumn{3}{c|}{ Designed Components } & \multicolumn{2}{c|}{ Simulation } & \multicolumn{2}{c|}{ \% Error } \\
\hline Design No. & $\boldsymbol{L}_{\boldsymbol{s}}(\boldsymbol{H})$ & $\boldsymbol{C}_{\boldsymbol{p}}(\boldsymbol{F})$ & $\boldsymbol{C}_{\boldsymbol{s}}(\boldsymbol{F})$ & $\boldsymbol{G}_{\boldsymbol{t r}}(\boldsymbol{V} \boldsymbol{V})$ & $\boldsymbol{f}_{\boldsymbol{r}}(\boldsymbol{k H z})$ & $\boldsymbol{G}_{t r}$ & $\boldsymbol{f}_{\boldsymbol{r}}$ \\
\hline $\mathbf{( 1 )}$ & $407 \mathrm{n}$ & $1.00 \mathrm{~m}$ & $100 \mathrm{u}$ & 0.535 & 25.0 & -2.7 & 0.0 \\
$\mathbf{( 2 )}$ & $58.2 \mathrm{u}$ & $142 \mathrm{n}$ & $28.4 \mathrm{n}$ & 0.599 & 125.2 & -0.2 & 0.2 \\
$\mathbf{( 3 )}$ & $2.00 \mathrm{u}$ & $900 \mathrm{n}$ & $1.80 \mathrm{u}$ & 0.700 & 100.1 & 0.0 & 0.1 \\
$\mathbf{( 4 )}$ & $447 \mathrm{u}$ & $333 \mathrm{n}$ & $333 \mathrm{n}$ & 0.778 & 15.1 & -2.8 & 0.6 \\
$\mathbf{( 5 )}$ & $59.8 \mathrm{~m}$ & $333 \mathrm{p}$ & $83.3 \mathrm{p}$ & 0.879 & 75.2 & -2.3 & 0.3 \\
$\mathbf{( 6 )}$ & $58.0 \mathrm{u}$ & $667 \mathrm{n}$ & $133 \mathrm{n}$ & 1.00 & 60.1 & 0.0 & 0.1 \\
$\mathbf{( 7 )}$ & $47.3 \mathrm{u}$ & $3.75 \mathrm{u}$ & $1.88 \mathrm{u}$ & 2.01 & 20.1 & 0.5 & 0.0 \\
$\mathbf{( 8 )}$ & $32.6 \mathrm{u}$ & $113 \mathrm{n}$ & $225 \mathrm{n}$ & 4.98 & 99.9 & -0.4 & -0.1 \\
$\mathbf{( 9 )}$ & $559 \mathrm{n}$ & $9.50 \mathrm{~m}$ & $475 \mathrm{u}$ & 9.98 & 20.0 & -0.2 & 0 \\
$\mathbf{( 1 0 )}$ & $35.7 \mathrm{u}$ & $3.12 \mathrm{u}$ & $312 \mathrm{n}$ & 20.1 & 50.0 & 0.5 & 0 \\
\hline
\end{tabular}

Substituting (9), (24), and (25) into (23) and recalling that $V_{C p_{M 1}}(0)=-N V_{o}$, gives

$$
Q=\frac{\pi\left(4 G_{\mathrm{tr}}\left((A+1) G_{\mathrm{tr}}-1\right)+1\right)}{4\left(2 G_{\mathrm{tr}}-1\right)} .
$$

It should be noted that the stored energy changes during each mode, as energy is first stored and then transferred to the load during respective modes. The effective " $Q$ " therefore changes during each mode. The "energy profile" in steady-state therefore consists of a dc-value upon which a triangular-type energy profile is imposed. For the analysis presented here, the dc-baseline value at the start of M1 is therefore used.

To consider the accuracy of the model, at resonance, various converter designs have been generated using (6), (22) to satisfy the specifications given in Table I. The resulting ideal component values, given in Table II, are used for simulating each converter in SPICE. Also given in Table II are the values of $G_{\mathrm{tr}}$ and $f_{r}$ obtained from the simulations, from which it can be seen that an excellent correlation between them exists.

\section{A. Load Dependent Tank Current}

To maintain high part-load efficiency, it is desirable that the tank current $\left(I_{L s}\right)$ reduces as $R_{L}$ increases. From (6):

$$
G_{\mathrm{tr}}-\frac{1}{2}=2 N^{2} R_{L} f_{r} C_{p}
$$

Since $f_{r}$ increases for increasing $R_{L}$, doubling $R_{L}$ results in at least a doubling of the r.h.s of (27). Note that with a near 
short-circuit load, $C_{p}$ is effectively short-circuited and the resonant frequency is given by the series tank components. Conversely, with an open-circuit output, the rectifier is effectively disconnected and $f_{r}$ is a function of both $C_{p}$ and $C_{\mathrm{s}}$, resulting in a higher resonant frequency. Consequently, if the r.h.s of (27) is initially large, doubling $R_{L}$ will result in $G_{\mathrm{tr}}$ approximately doubling, thereby requiring operation well above resonance to regulate the output voltage. The resulting $I_{L s}$ will therefore be much larger than necessary. Conversely, if the r.h.s of (27) is made relatively small, for the minimum $R_{L}$, providing $f_{r}$ is only modified slightly with an increase in resistance, $G_{\text {tr }}$ will remain approximately constant, and operation can remain close to resonance. This implies two conditions for good loading performance. First, $A$ should be chosen to be large to provide good resonant frequency stability, and secondly, $G_{\mathrm{tr}}$ should be chosen to be $\approx 1 / 2$ for the maximum load (minimum $R_{L}$ ). The penalty for this, is that, from (22) a higher value for $L_{s}$ is required, and from (26) a larger resonant $Q$.

\section{B. Real Power Transfer by Harmonic Excitation}

For applications where only very limited changes in load are expected (in some lighting applications, for instance) and a regulated output voltage is required, it is now shown that it is possible to reduce the peak current stresses on the tank components using harmonic power transfer. Considering (14) and (19) which describe the tank current, the peak can occur in either M1 or M2. If the derivative of $I_{L s}$ at the end of M1, is positive, i.e., $V_{L s_{M 2}}(0)>0$, see (17), the peak current occurs in M2. Conversely, if the gradient is negative, the current has already peaked during M1. From (14), (19) the peak current is obtained as (28), shown at the bottom of the page.

For a particular converter operating at high efficiency at resonance, with a $50 \%$ duty cycle, $I_{\mathrm{Ls}}$ can be assumed sinusoidal, and all power supplied to the converter is real. Therefore to a first approximation the input and output power can be equated

$$
P_{\mathrm{in}}=\left(\frac{V_{i}}{2}\right)\left(\frac{2 \hat{I}_{p}}{\pi}\right), P_{\mathrm{out}}=\frac{\left(V_{i} G_{\mathrm{tr}}\right)^{2}}{N^{2} R_{L}}, \quad P_{\mathrm{in}}=P_{\mathrm{out}} .
$$

Simple rearrangement leads to $\hat{I}_{p}$ being given by

$$
\hat{I}_{p}=\frac{\pi V_{i} G_{\mathrm{tr}}^{2}}{N^{2} R_{L}}
$$

where $\hat{I}_{p}$ corresponds to the minimum sinusoidal current peak that can be achieved for a given design specification. Using (28), (30) gives

$$
\hat{I}_{L s_{\mathrm{norm} 1}}=\left(\frac{\hat{I}_{L s}-\hat{I}_{p}}{\hat{I}_{p}}\right)
$$

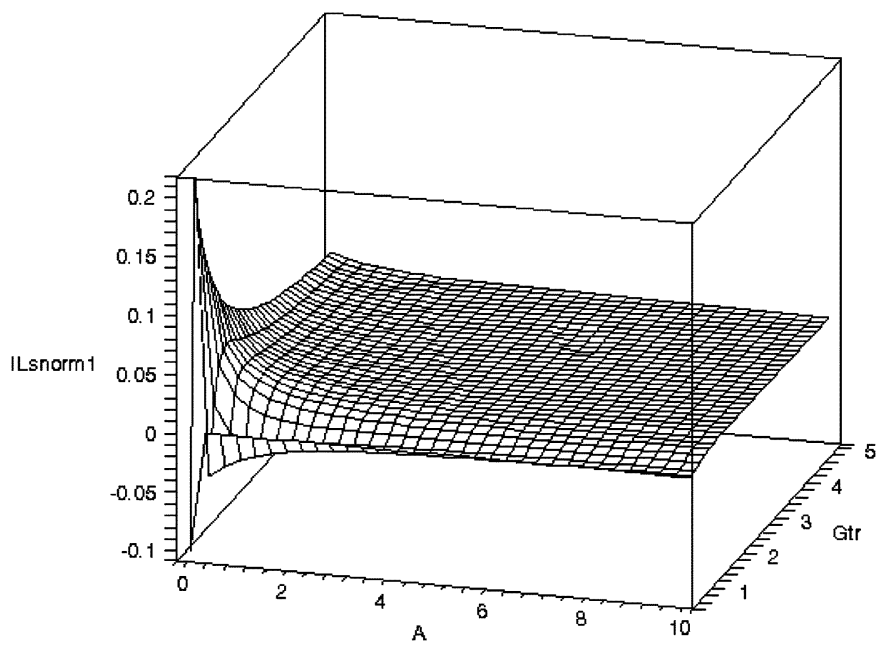

Fig. 4. 3-D plot of normalized inductor current at resonance.

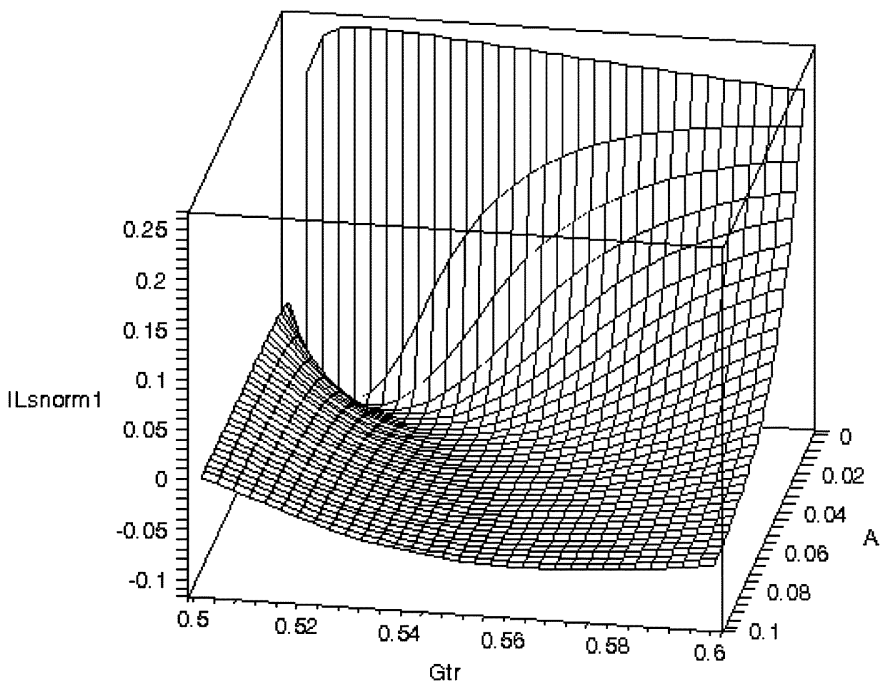

Fig. 5. 3-D plot of normalized inductor current at resonance in the low $G_{\mathrm{tr}}$ and low $A$ regions.

where $\hat{I}_{L s_{\text {norm } 1}}$ is the peak current normalized to an equivalent sinusoid, at resonance, and from (15), (20), (22), (28), and (30) is solely a function of $A$ and $G_{\mathrm{tr}}$.

A 3-D plot showing the characteristic of (31) is given in Fig. 4, from where it can be seen that $\hat{I}_{\mathrm{Ls}}$ tends to the value described by (30), i.e., (31) tends to zero. It can also be seen that in certain regions of the $A, G_{\mathrm{tr}}$ plane, significant variations in current stress is evident.

For completeness, Fig. 5 provides a magnified view of Fig. 4 in the region of low $G_{\mathrm{tr}}$ and low $A$.

It is therefore clear that a region exists where $\hat{I}_{L s}$ is lower than would be expected if sinusoidal tank currents were induced, thereby indicating that significant real power is being transferred

$$
\hat{I}_{L s}=\frac{V_{i}}{8 N \sqrt{R_{L} f_{r} L_{s}\left(2 G_{\mathrm{tr}}-1\right)}}\left(\begin{array}{c}
\frac{\left(\operatorname{sgn}\left(4 A G_{\mathrm{tr}}^{2}-4 A G_{\mathrm{tr}}+4 G_{\mathrm{tr}}^{2}-4 G_{\mathrm{tr}}+1\right)+1\right)\left(4 A G_{\mathrm{tr}}^{2}+4 G_{\mathrm{tr}}^{2}-1\right)}{\sqrt{A+1}} \ldots \\
+\frac{\left(1-\operatorname{sgn}\left(4 A G_{\mathrm{tr}}^{2}-4 A G_{\mathrm{tr}}+4 G_{\mathrm{tr}}^{2}-4 G_{\mathrm{tr}}+1\right)\right)\left|4 A G_{\mathrm{tr}}^{2}+4 G_{\mathrm{tr}}^{2}-4 G_{\mathrm{tr}}+1\right|}{\sqrt{A}}
\end{array}\right)
$$




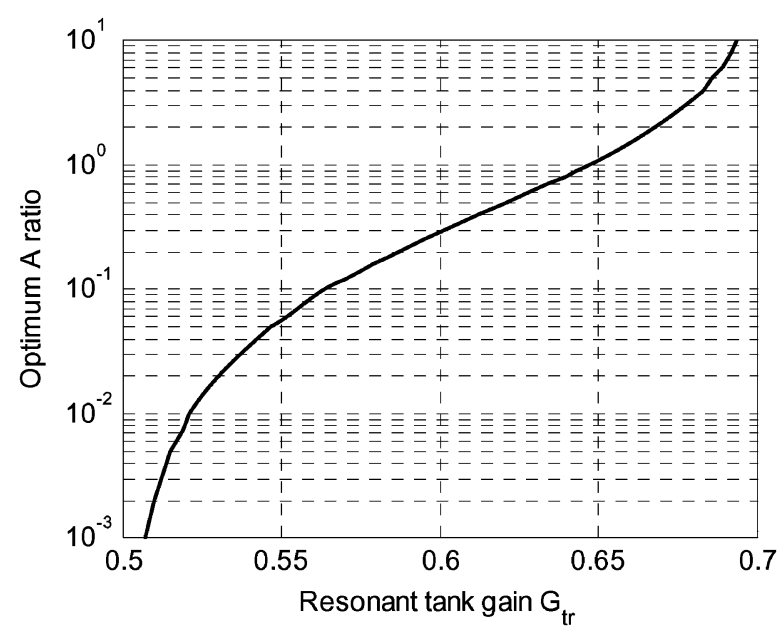

Fig. 6. Optimum $A$ for peak current reduction versus $G_{\operatorname{tr}}$.

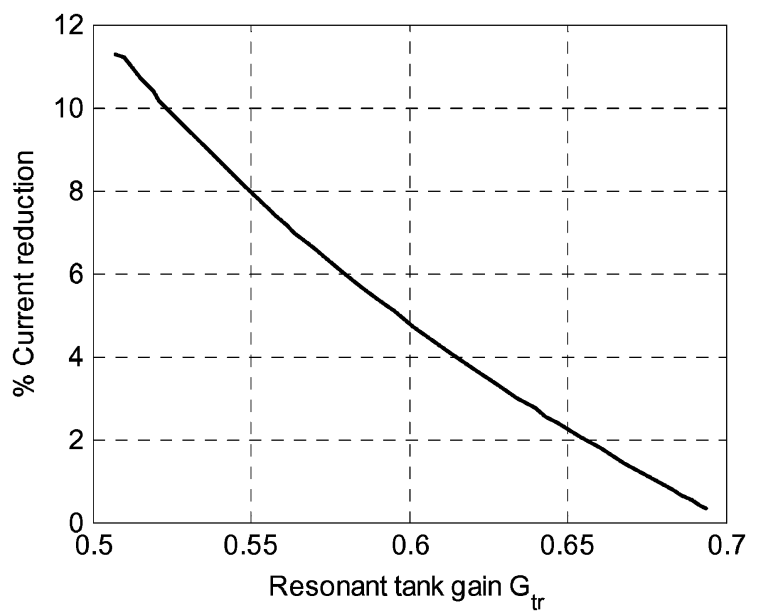

Fig. 7. Current reduction at optimum $A$ versus $G_{\text {tr }}$.

on harmonics. Also, from Fig. 5 it can be seen that as $G_{\text {tr }}$ is varied, the minimum peak current occurs at a particular value of $A$ that can be numerically calculated. The result is given in Fig. 6. The corresponding percentage reduction in peak tank current is plotted in Fig. 7.

Again, it can be seen that the penalty for reducing the current stresses on the tank components is that $A$ is small, thereby incurring a large variation in resonant frequency as the load changes, and reducing part-load efficiency. This design principle is therefore preferred when load variations are expected to be small.

\section{Impact of Component Value Choice}

From (6)

$$
C_{p}=\frac{F_{1}\left(G_{\mathrm{tr}}\right)}{N^{2} R_{L} f_{r}}
$$

and the required series inductor from (22) can be written

$$
L_{s}=\frac{N^{2} R_{L}}{f_{r}} F_{2}\left(A, G_{\mathrm{tr}}\right) \text {. }
$$

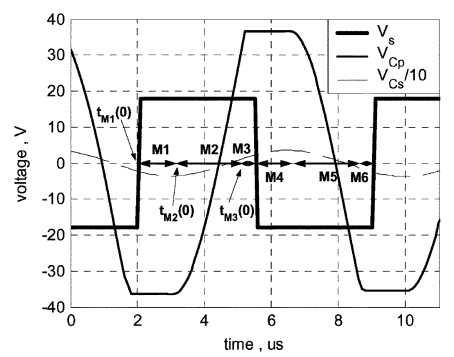

(a)

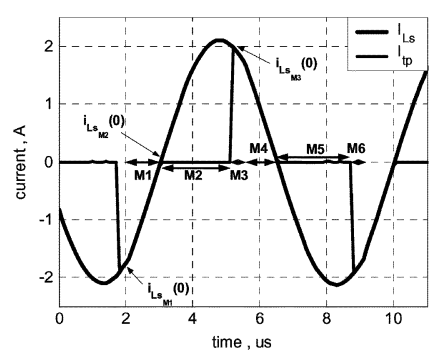

(b)
Fig. 8. Modes during above resonance operation of a heavily loaded converter: (a) voltage waveforms and (b) current waveforms.

Equating $f_{r}$ terms in both (32) and (33) provides the relationship

$$
\frac{L_{s}}{4 N^{4} R_{L}^{2} C_{p}}=\frac{F_{2}\left(A, G_{\mathrm{tr}}\right)}{F_{1}\left(G_{\mathrm{tr}}\right)}
$$

and equating the $N^{2} R_{L}$ terms in both (32) and (33) gives

$$
f_{r}=\frac{1}{2} \sqrt{\frac{F_{1}\left(G_{\mathrm{tr}}\right) F_{2}\left(A, G_{\mathrm{tr}}\right)}{L_{s} C_{p}}}
$$

Equations (34) and (35) provide important insight into the impact of component and parameter choices that are available to the designer. It will ultimately be shown that converters with similar values of $A$ and $G_{\mathrm{tr}}$ possess similar properties, such as their normalized frequency responses. Moreover, it may be desirable to maintain certain favourable properties of a previously used design, with a new specification of converter. The following section therefore demonstrates how one can simply change 1) the minimum load, 2) the overall gain, and 3) the resonant frequency, of an existing design, to accommodate a different application requirement.

Scenario 1-From (34) it can be seen that the converter's component values can be varied while maintaining a specific $G_{\text {tr }}$ by ensuring that both the l.h.s of (34) and $A$ remain constant. This implies that $R_{L}$ can, for example, be doubled and $G_{\mathrm{tr}}$ maintained providing $N$ remains constant, and $L_{s} / C_{p}$ quadrupled. However, in general, this will result in a change of $f_{r}$. If this is not desired, maintaining $f_{r}$, from (35), requires a constant $C_{p} L_{s}$ product. Doubling $L_{s}$ and halving $C_{p}$ is therefore necessary.

Scenario 2-Alternatively, it may be desirable that the overall converter gain $\left(G_{\mathrm{tr}} / N\right)$ be doubled, for instance. The most straightforward method to achieve this is via $N$. While maintaining $G_{\mathrm{tr}}, N$ simply has to be halved. However, since $A$ and $G_{\mathrm{tr}}$ are to remain constant, the resonant tank components must be appropriately selected i.e., from (34) $L_{s} / C_{p}$ must reduce by a factor of 16 , generally resulting in a change of resonant frequency. Again, if this is not desirable, maintaining the resonant frequency, from (35), requires a constant $C_{p} L_{s}$ product. Consequently, $C_{p}$ has to be quadrupled and $L_{s}$ quartered.

Scenario 3-Changing $f_{r}$, while maintaining $N, G_{\mathrm{tr}}$, and $A$, for a nominal load, is achieved through the appropriate choice of tank component values. From (34) and (35) doubling $f_{r}$ is achieved by halving both $L_{s}$ and $C_{p}$. Equation (34) is also useful when considering the conversion from ideal to preferred component values, since the gain is usually the determining factor in a converter design. From (34) the preferred values can be chosen 


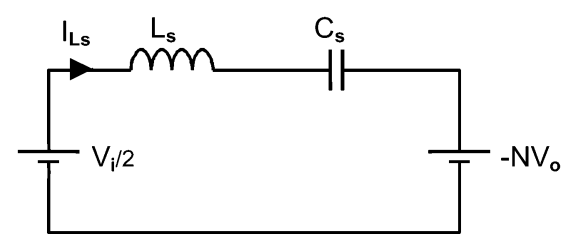

(a)

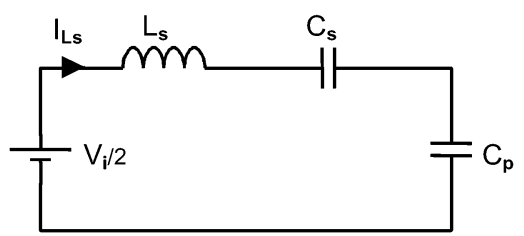

(b)

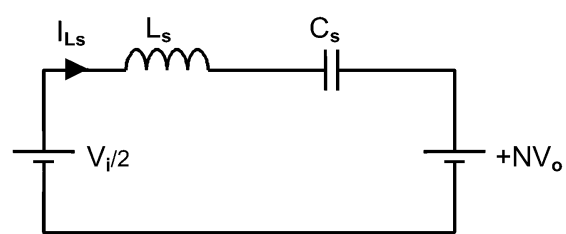

(c)

Fig. 9. Equivalent circuits for (a) M1, (b) M2, and (c) M3 for a heavily loaded converter operating above resonance.

to satisfy component value ratio constraints, such as $L_{s} / C_{p}$ and $A$, giving greater flexibility.

\section{HEAVILY LOADED OPERATION $\left(1 \leq f_{m} \leq\right.$ $\left.\left(2 G_{O}+1\right) /\left(2 G_{\mathrm{tr}}-1\right)\right)$}

Fig. 8 shows typical current and voltage waveforms for converter operation above resonance. The behavior can be divided into 6 modes (M1, M2 ...M6). Due to symmetry, it is sufficient to restrict the analysis to the first half cycle of operation.

Equivalent circuits for M1, M2, and M3 are given, respectively, in Fig. 9 (a)-(c), and can be used to form a piecewise steady-state solution of the converter's behavior.

Some fundamental definitions are

$$
\begin{aligned}
V_{o} & =\frac{G_{o}}{N} V_{i} \\
f_{s} & =f_{m} f_{r}
\end{aligned}
$$

where $f_{r}$ is the resonant frequency, $f_{s}$ is the switching frequency, $f_{m}>1$ for operation above resonance and $G_{o}$ is the resonant tank gain at $f_{s}$. It is important to consider the power transfer mechanisms in the converter. For high efficiency operation, as a first approximation, $P_{s}, P_{t w}$ and $P_{o}$, are considered equal. From Fig. 8, the following expressions can be written, where $t_{M 1}, t_{M 2}, t_{M 3}$ are the total times spend in M1, M2, M3, respectively

$$
\begin{aligned}
P_{s} & =V_{i} f_{m} f_{r}\left(Q_{M 1}+Q_{M 2}+Q_{M 3}\right) \\
P_{t w} & =2 G_{o} V_{i} f_{m} f_{r}\left(-Q_{M 1}+Q_{M 3}\right), P_{s} \approx P_{t w} \approx P_{o} \\
P_{o} & =\frac{V_{i}^{2} G_{o}^{2}}{N^{2} R_{L}} .
\end{aligned}
$$

Also from Fig. 8, it can be seen that no power transfer from the supply to the load occurs during M2 since no current enters the primary of the transformer.

During M2 all the tank current charges $C_{p}$ from $-N V_{o}$ to $+N V_{o}$, then, when (5) is used as a substitution for $C_{p}$ the total charge is given as

$$
Q_{M 2}=\frac{V_{i} G_{o}\left(2 G_{\mathrm{tr}}-1\right)}{2 f_{r} N^{2} R_{L}}
$$

Since the procedure to calculate the mode times for heavy loading is similar to that used previously, when the converter is operating at resonance, the derivation and mode time expressions are summarized in Appendix A.

\section{LightLY LOADED CONVERTER $\left(f_{m} \geq\left(2 G_{o}+1 / 2 G_{\text {tr }}-1\right)\right)$}

The methodology presented in this section is similar to that for heavy loading. Fig. 10 shows example current and voltage

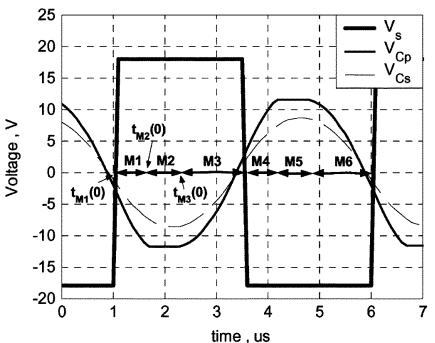

(a)

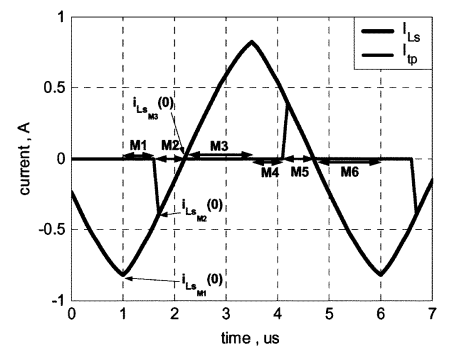

(b)
Fig. 10. Modes of operation of a lightly loaded converter: (a) voltage waveforms and (b) current waveforms.

waveforms for operation above resonance under light loading conditions. The behavior of the converter can be divided into 6 modes (M1, M2 ... M6). Once again, it is sufficient to analyse the converter during the first half cycle only.

Equivalent circuits for M1,M2,M3 are given in Fig. 11(a)-(c), respectively.

From Fig. 10, the following expressions for $P_{s}, P_{t w}$ and $P_{o}$ can be derived (note that all power transfer to the load occurs during M2):

$$
\begin{aligned}
P_{s} & =V_{i} f_{m} f_{r}\left(Q_{M 1}+Q_{M 2}+Q_{M 3}\right) \\
P_{t w} & =-2 G_{o} V_{i} f_{m} f_{r} Q_{M 2}, \quad P_{s} \approx P_{t w} \approx P_{o} \\
P_{o} & =\frac{V_{i}^{2} G_{o}^{2}}{N^{2} R_{L}} .
\end{aligned}
$$

During M3, M4 all the tank current charges $C_{p}$ from $-N V_{o}$ to $+N V_{o} \mathrm{~V}$, and the following relationship is obtained when (5) is used as a substitution for $C_{p}$ :

$$
\frac{V_{i} G_{o}\left(2 G_{\mathrm{tr}}-1\right)}{2 f_{r} N^{2} R_{L}}=-Q_{M 1}+Q_{M 3} .
$$

Since the procedure to calculate the mode times for light loading is similar to that used previously, when the converter is operating at resonance, the derivation and mode time expressions are summarized in Appendix B.

\section{NORMALIZED FREQUENCY RESPONSE}

During steady-state operation, the half-cycle switching period is the sum of the mode times

$$
t_{M 1}+t_{M 2}+t_{M 3}-\frac{1}{2 f_{m} f_{r}}=0 .
$$

The expressions for the mode times given in the Appendices, equations $\{(\mathrm{A} 9),(\mathrm{A} 12),(\mathrm{A} 17)\}$ and $\{(\mathrm{A} 27),(\mathrm{A} 32),(\mathrm{A} 39)\}$, can be substituted into (42) to provide the half-cycle solution 


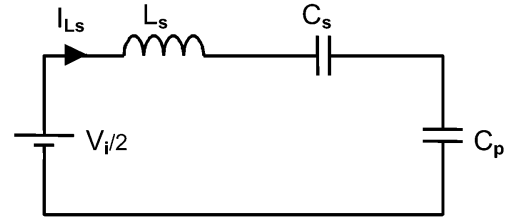

(a)

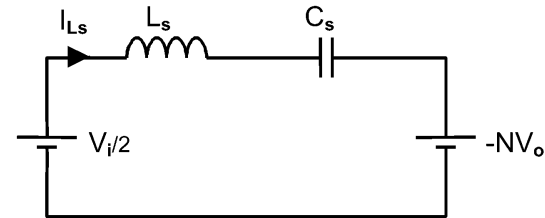

(b)

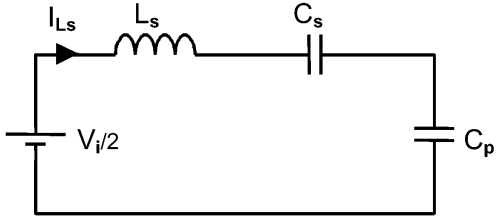

(c)

Fig. 11. Equivalent circuits during modes: (a) M1, (b) M2, and (c) M3 for a lightly loaded converter operating above resonance.

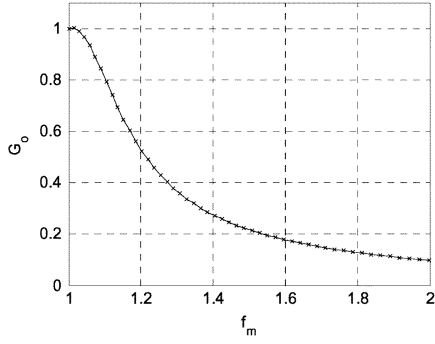

(a)

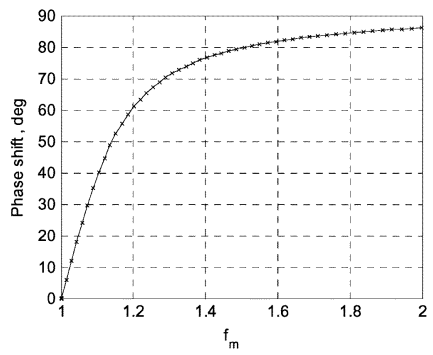

(b)
Fig. 12. Tank frequency response: (a) magnitude and (b) phase response for $G_{\mathrm{tr}}=A=1$.

for the heavily and lightly loaded cases, respectively. When $L_{s}$ is eliminated from the mode time expressions through the use of (15), (20) and (22), the converter frequency response in (42) is explicitly dependent on only four variables viz. $G_{\mathrm{tr}}, A, G_{o}, f_{m}$.

Equation (42) can be used to generate a frequency response to aid in the design of a converter. For example, a designer may choose to nominally operate at a specific percentage above $f_{r}$, with a given $G_{O}$ and $A$, the only unknown then being $G_{\mathrm{tr}}$. Once a particular $G_{\mathrm{tr}}$ is obtained, a converter design can be simply generated from (2), (6), (7), (15), (20), and (22).

While generating a frequency response it is necessary to identify whether the converter is heavily or lightly loaded so that the correct set of equations can be utilized. This is achieved through analysis of the mode times. At the transition between the two conditions, when the converter is critically loaded, $t_{M 3 \text { (heavy loading) }}=t_{M 1 \text { (light loading) }}=0$. Equating the charge transfer through the series inductor during either of these two modes, to zero (A2), (A18), and solving for $f_{m}$ provides the following inequality for heavy loading operation (note that using mode time expressions (A17), (A39) also leads to the same result, although the calculation is more complex):

$$
f_{m}<\frac{2 G_{o}+1}{2 G_{\mathrm{tr}}-1} .
$$

Hence, if heavy loading is initially assumed, after solving $f_{m}$ for a given $G_{O}$ and $G_{\mathrm{tr}}$, if (43) is satisfied then the heavy loading assumption is valid. Interestingly, setting $t_{\mathrm{M} 3}=0$ in the heavy loading condition of (42) and utilizing (22) and (43) to eliminate $L_{s}$ and $f_{m}$, respectively, provides an expression relating $G_{o}$ directly to $G_{\mathrm{tr}}$ at the critical loading point.

As an example of computation overhead, Fig. 12 shows (a) the resonant tank frequency response and (b) phase response, for $G_{\mathrm{tr}}=A=1$ - the results show 50-point curves that were calculated in 2 s on a P4 $2.4 \mathrm{GHz}$, running MATLAB.

\section{NORMALIZED CONVERTER-OUTPUT REGULATION}

The behavior of the converter, as a function of the load, is now investigated. For convenience, the applied load, $R_{L}$, and the minimum load, $R_{O}$, are related by the load scaling factor, $R_{x}$, as follows:

$$
R_{L}=R_{o} R_{x}
$$

From (6), (22), expressions for $G_{\mathrm{tr}}$ and $f_{r}$ can be obtained by virtue of the resonant tank components remaining unchanged as the load is varied (where $G_{\text {tro }}, f_{\text {ro }}$ are the resonant tank gain and resonant frequency, respectively, when the load is at its nominal value $\left.\left(R_{x}=1\right)\right)$. From (6)

$$
\begin{aligned}
C_{p}=\frac{F_{1}\left(G_{\mathrm{tro}}\right)}{N^{2} R_{o} f_{\mathrm{ro}}}=\frac{F_{1}\left(G_{\mathrm{tr}}\right)}{N^{2} R_{x} R_{o} f_{r}} \\
\therefore \quad \frac{f_{r}}{f_{\mathrm{ro}}}=\frac{F_{1}\left(G_{\mathrm{tr}}\right)}{R_{x} F_{1}\left(G_{\mathrm{tro}}\right)}=\frac{\left(2 G_{\mathrm{tr}}-1\right)}{\left(2 G_{\mathrm{tro}}-1\right) R_{x}} .
\end{aligned}
$$

Similarly, from (22) and using (45) to eliminate $f_{r} / f_{\text {ro }}$ gives

$$
\begin{aligned}
& \frac{L_{s} f_{r}}{N^{2} R_{o}}=\frac{f_{r}}{f_{\mathrm{ro}}} F_{2}\left(A, G_{\mathrm{tro}}\right)=R_{x} F_{2}\left(A, G_{\mathrm{tr}}\right) \\
& \rightarrow \sqrt{\frac{F_{2}\left(A, G_{\mathrm{tro}}\right)}{F_{1}\left(G_{\mathrm{tro}}\right)}}=R_{x} \sqrt{\frac{F_{2}\left(A, G_{\mathrm{tr}}\right)}{F_{1}\left(G_{\mathrm{tr}}\right)}}
\end{aligned}
$$

and

$$
\begin{aligned}
& R_{x}=\sqrt{\frac{F_{2}\left(A, G_{\mathrm{tro}}\right) F_{1}\left(G_{\mathrm{tr}}\right)}{F_{1}\left(G_{\mathrm{tro}}\right) F_{2}\left(A, G_{\mathrm{tr}}\right)}}=\frac{F_{3}\left(A, G_{\mathrm{tr}}\right)}{F_{3}\left(A, G_{\mathrm{tro}}\right)} . \\
& \text { where } F_{3}\left(A, G_{\mathrm{tr}}\right)=\sqrt{\frac{F_{1}\left(G_{\mathrm{tr}}\right)}{F_{2}\left(A, G_{\mathrm{tr}}\right)}} .
\end{aligned}
$$

Since (47) is dependent only on $G_{\mathrm{tr}}, G_{\mathrm{tro}}, R_{x}$ and $A$, a function $F_{4}$ numerically exists which satisfies the following:

$$
\begin{aligned}
G_{\mathrm{tr}}-F_{4}\left(G_{\mathrm{tro}}, R_{x}, A\right)=R_{x} F_{3}\left(A, G_{\mathrm{tro}}\right) & \\
& -F_{3}\left(A, G_{\mathrm{tr}}\right)=0 .
\end{aligned}
$$

From (48), as $R_{x}$ is varied, the change in $G_{\mathrm{tr}}$, and hence, $f_{r} / f_{\text {ro }}$ [see (45)] is identical for converters with the same $A$ and $G_{\text {tro }}$ at nominal load. In (42) it was also established that the frequency response of a converter is fully defined by its $G_{\operatorname{tr}}$ and $A$, hence, as load is varied, for a given $A$ and $G_{\text {tro }}$ at nominal load, the converter's normalized frequency response for all values of $R_{x}$ is also fully defined, thereby implying that the normalized regulation performance of converters with similar $G_{\text {tro }}$ and $A$, are similar. Consequently, assuming high efficiency operation, it is not necessary to analyse cases on an individual basis, even if respective converters differ in $f_{\text {ro }}, N$, and $R_{O}$. 


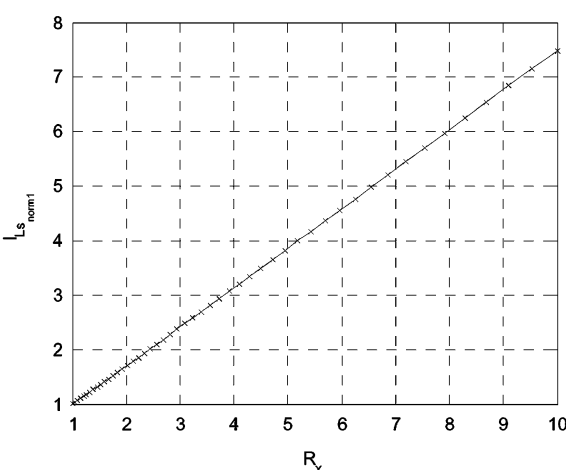

(a)

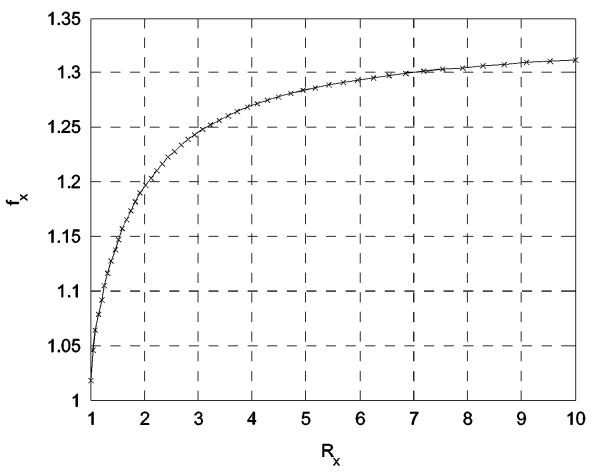

(d)

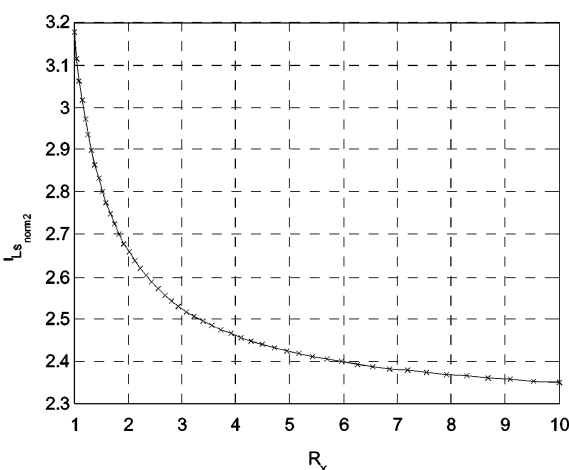

(b)

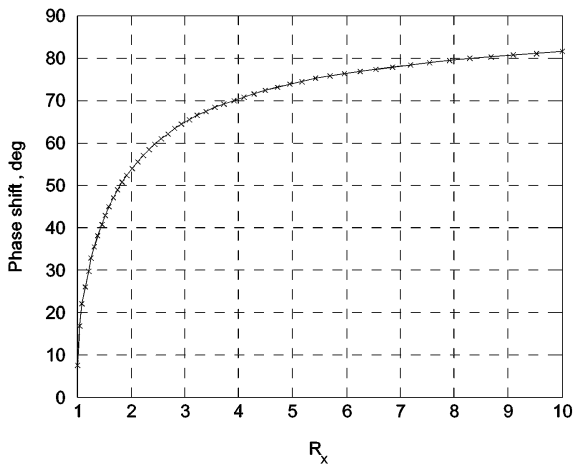

(e)

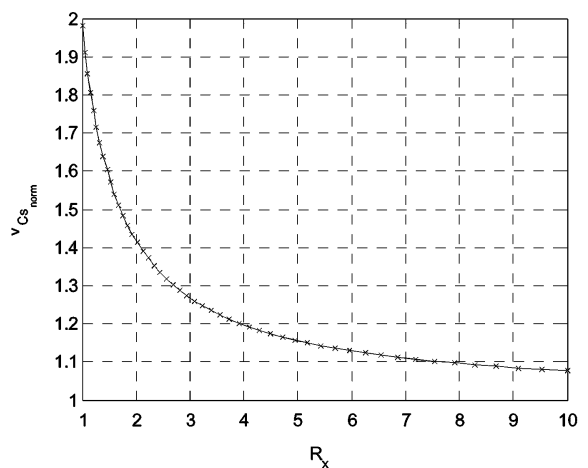

(c)

Fig. 13. (a), (b), (c), (d), and (e) provide the regulation performance curves for $G_{\text {tro }}=A=1$. (a) Peak resonant current normalized to a sinusoid at resonance $\left(\hat{I}_{L s}=I_{L s_{\text {norm } 1}}\left(V_{i} / N^{2} R_{o}\right)\left(\pi G_{o}^{2} / R_{x}\right)\right)$. (b) Peak resonant current normalized to nominal load $\left(\hat{I}_{L s}=I_{L s_{\text {norm } 2}}\left(V_{i} / N^{2} R_{o}\right)\right)$. (c) Peak series capacitor voltage stress $\left(\hat{V}_{C s}=V_{C s_{\text {norm }}} V_{i}\right)$. (d) Normalized Switching frequency wrt nominal load $\left(f_{s}=f_{x} f_{\mathrm{ro}}\right)$. (e) Phase shift required for output regulation.

It is notable, however, that when the converter is regulated above resonance at nominal load and $G_{0}>1 / 2$ (the normal condition), performance curves for $G_{\mathrm{tro}}=G_{0}$ can be used, since the converter can be thought to be operating with $R_{\mathrm{Lmin}}>$ $R_{o}$. For $G_{0}<1 / 2$ this analogy cannot be used since converters cannot be generated with $G_{\mathrm{tr}} \leq 1 / 2$ at any load.

Fig. 13 provides the regulation performance curves for $G_{\text {tro }}=A=1$ (the 50-point curves are calculated in $2.5 \mathrm{~s}$ on a P4 $2.4 \mathrm{GHz}$ running MATLAB). Fig. 13(a) is particularly useful when assessing the part load efficiency of converters. Ideally, $I_{L s_{\text {norm } 1}}$ would remain at, or below, unity for all loads, since this corresponds to a sinusoidal current at resonance. In the example provided, when $R_{x}=10, \hat{I}_{\mathrm{Ls}}$ is 7.5 times larger than for a sinusoidal current at resonance, see (30). Therefore, improved part-load efficiency occurs at lower gradients on the plot.

Fig. 14 provides an example frequency response for $G_{\mathrm{tr}}=$ $A=1$. When generating the frequency response, if $f_{m}$ is numerically calculated for a given $G_{o}$, care must be taken since two solutions are present. From a practical perspective it is necessary that the gradient of the transfer function remains - ve (for operation above resonance) over the whole load range, thereby ensuring that positive feedback at the controller never occurs. In the example considered, operating below $f_{m} \approx 1.01$ may result in instability. In a practical converter, the effects of parasitics may eliminate this potential problem, or indeed accentuate it.

Converter operation has been previously described with respect to being either heavily or lightly loaded, the transition between the two being a critical point for analysis. While gener-

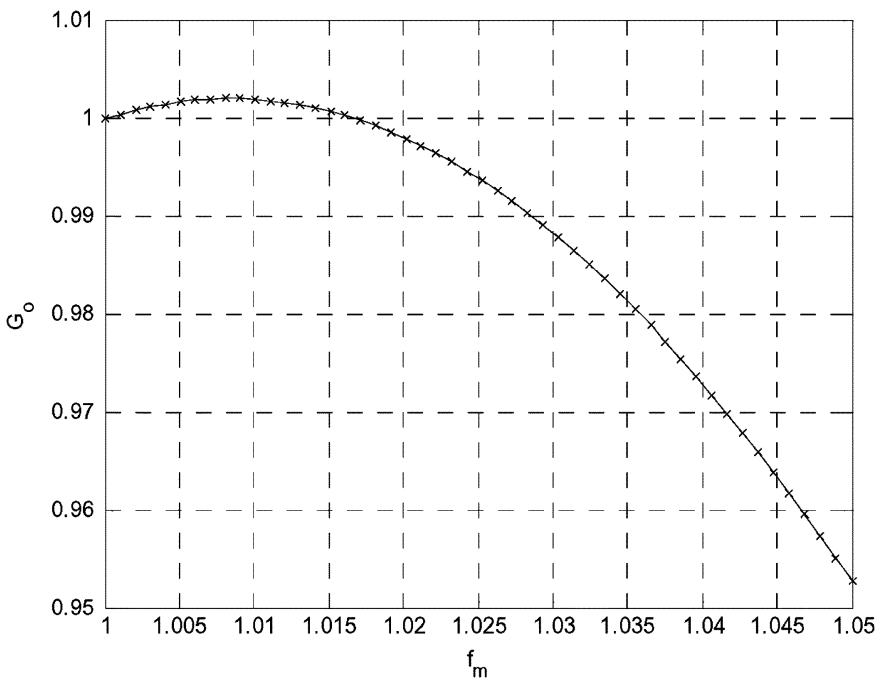

Fig. 14. Frequency response showing peak gain occurring above resonance.

ating the frequency response it has been necessary to find an expression for this borderline condition, see (43). In a similar manner, it is now also possible to derive an expression for the regulatory behavior of the converter (i.e., maintaining $G_{O}$ while varying $R_{L}$ through control of $f_{s}$ ). If it is assumed that the converter $G_{O}$ is regulated at $G_{\mathrm{tro}}$, as the load resistance is increased operation passes through the critical loading point into light loading. Setting $t_{M 3}=0$ in the heavy loading condition 


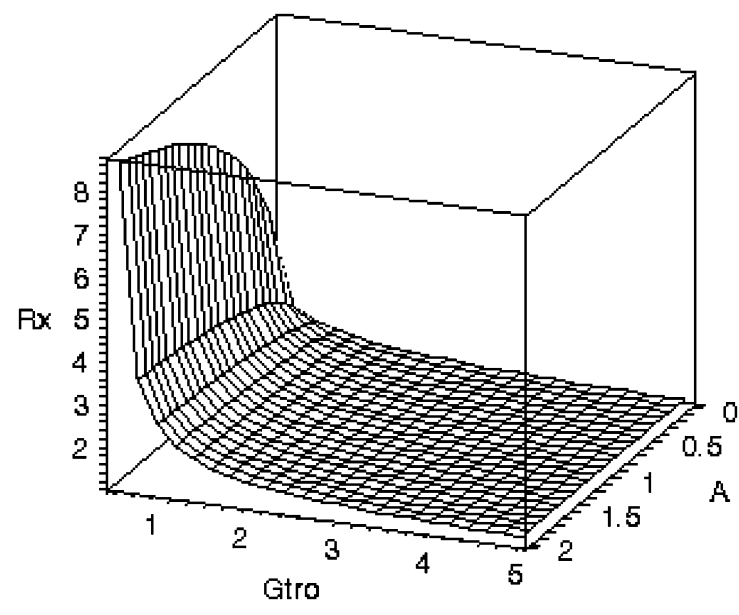

Fig. 15. Critical loading point $\left(R_{x}\right)$ for converter regulated at nominal resonant tank gain $G_{\text {tro }}$ and given $A$.

of (42) and utilizing equations (43), (44), (45) gives an analytical expression relating $R_{x}$ to $G_{\text {tro }}$ and $A$. Fig. 15 provides a plot of the resulting expression. From Fig. 15, for instance, it can be seen that when a converter is regulated about its nominal resonant tank gain of $G_{\text {tro }}=1$ and $A=2, R_{L}$ can increase by a factor of 2.7 from its nominal level prior to light loading occurring. Also from Fig. 15 it can be seen that for $G_{\text {tro }}<1$, the heavy loading condition dominates the characteristic, and that light loading is more prevalent for lower values of $A$ and large $G_{\text {tro. }}$.

\section{Normalized Converter Design CuRves}

Here, normalized design curves for several $G_{\text {tr }}$ values (see Fig. 16) are presented for the common case $A=1$. From the curves, the converter's magnitude and phase response can be obtained, and additionally component values and electrical component stresses derived. Finally, a degree of insight into the steady state bandwidth of the converter is also identified. note $G_{\mathrm{tr}}=\hat{G}_{o}$ for each curve.

To consider the steady-state regulation of the converter, $G_{\mathrm{tr}}$ should be considered as a function of load. The resonant frequency can then be calculated from (45). Once these parameters are known, Fig. 16(a) allows the necessary value of $f_{m}$ to maintain output voltage regulation, to be calculated, and subsequently, the associated switching frequency.

Fig. 16(e) provides the peak series inductor current stress. The 'low gradient' asymptote added to the graph provides the peak inductor current stress that would be obtained had the current been a sinusoid at resonance, see (30). This is useful when considering converter efficiency.

From (48) it can be seen that $F_{3}\left(A, G_{\mathrm{tr}}\right)=R_{x} F_{3}\left(A, G_{\mathrm{tro}}\right)$. Since $G_{\text {tro }}$ at $R_{L}=R_{o}$ is well defined, $F_{3}\left(A, G_{\text {tro }}\right)$ can be readily evaluated. Now, if $R_{L}$ is doubled from the nominal value $\left(R_{x}=2\right)$, for example, the converter $G_{\mathrm{tr}}$ will vary. The value of $G_{\mathrm{tr}}$ obtained after this doubling can most easily be found graphically. Fig. $16(\mathrm{~g})$ provides a plot of $F_{3}\left(A, G_{\mathrm{tr}}\right)$ for $A=1$.

Fig. 16(h) provides the necessary filter capacitance $\left(C_{f}\right)$ to obtain a certain percentage output voltage ripple. During periods when $C_{p}$ is charging, the output current is maintained by the charge stored on $C_{\mathrm{f}}$. Assuming a constant load current implies a linear reduction in output voltage, which can be calculated from knowledge of the mode times.

From Fig. 16(a) it can be seen that as $G_{\text {tr }}$ increases, the necessary $f_{m}$ for output voltage regulation is bounded. The highest value of $f_{m}$ occurs during light loading, when $G_{\text {tr }}$ is high. Finding the limit of (42) for light loading, as $G_{\mathrm{tr}} \rightarrow \infty$ and solving for $f_{m}$ gives the following:

$$
f_{m_{\max }} \rightarrow \frac{\pi}{2 \cos ^{-1}\left(\frac{1}{1+2 G_{o}(A+1)}\right)} .
$$

As the load tends to open circuit the resonant frequency tends to

$$
f_{r_{\max }} \rightarrow \frac{1}{2 \pi} \sqrt{\frac{A+1}{L_{s} C_{p}}} .
$$

Equations (49) and (50) can therefore be used to calculate the theoretical maximum steady-state control bandwidth to regulate the converter from nominal load to open circuit. The peak switching frequency required to regulate the converter tank gain at $G_{o}=G_{\text {tro }}$ occurs at $f_{s_{\max }}=f_{m_{\max }} f_{r_{\max }}$ when the load resistance is assumed infinite. If $G_{0}>1 / 2$, which is the usual case, then the minimum switching frequency required to regulate the converter output voltage occurs at resonance when the load is at its nominal load. Solving (6) for $R_{L}$ and substituting this into (22) provides an $L_{s} C_{p}$ product. Substituting this product into the expression for $f_{s_{\max }}$ and dividing by $f_{r}$ provides a measure of the steady-state control bandwidth dependent on $G_{\text {tr }}$ at the nominal load $\left(G_{\text {tro }}\right)$ and $A$ only. Fig. 17 provides a measure of the steady-state bandwidth (maximum frequency/minimum frequency) as $G_{\text {tro }}$ is varied, for several $A$ values ranging from 0.2 to 2 in steps of 0.2 . Note that for $A=1$ the maximum value is 1.8 .

\section{Design EXAMPLE With EXPERIMENTAL Results}

The use of the design curves in Fig. 16 will now be demonstrated by example. A converter specification is given in Table III, from where it can be seen that the specification includes rectifier on-state voltages that can be modelled by parasitic load resistors, effectively making the overall load resistance appear larger. The overall gain is therefore reduced due to the potential divider network that is then present. The following expressions can be used to compensate for the rectifier voltage drop: (where $R_{L}$ ' and $G_{o}$ ' are the required effective load resistance and tank gain to achieve the specifications)

$$
\begin{aligned}
& R_{L}^{\prime}=R_{L}\left(1+\frac{2 V_{d}}{V_{o}}\right) \\
& G_{o}^{\prime}=G_{o}\left(1+\frac{2 V_{d}}{V_{o}}\right) .
\end{aligned}
$$

For simplicity the design process can be subdivided into convenient steps: 


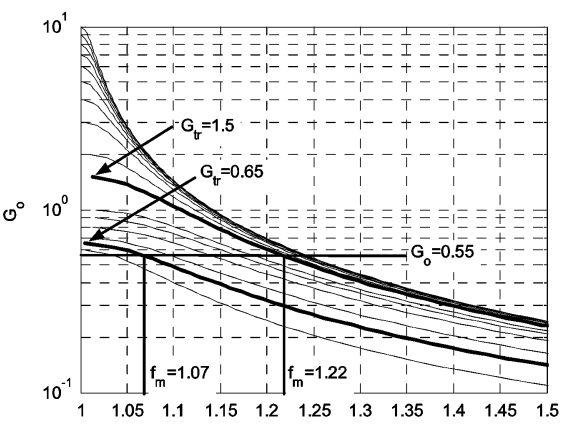

$\mathrm{f}_{\mathrm{m}}$

(a)

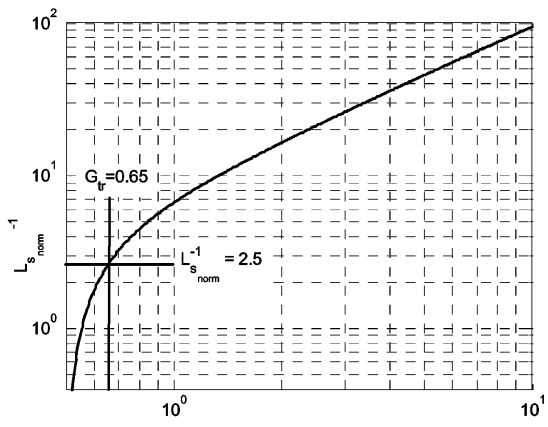

$\mathrm{G}_{\mathrm{tr}}$

(d)

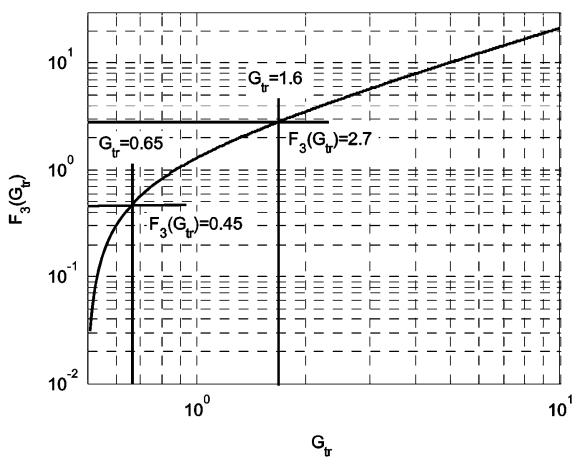

(g)

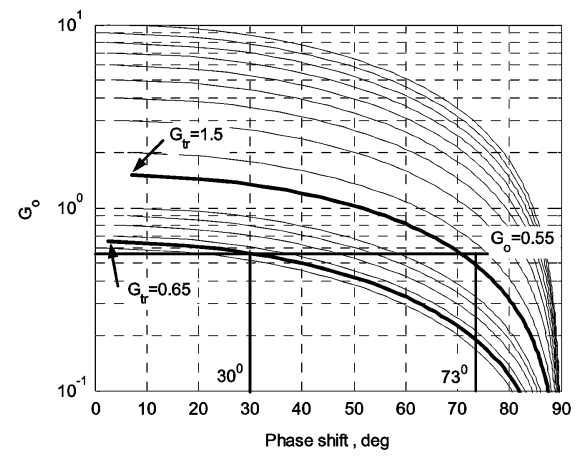

(b)

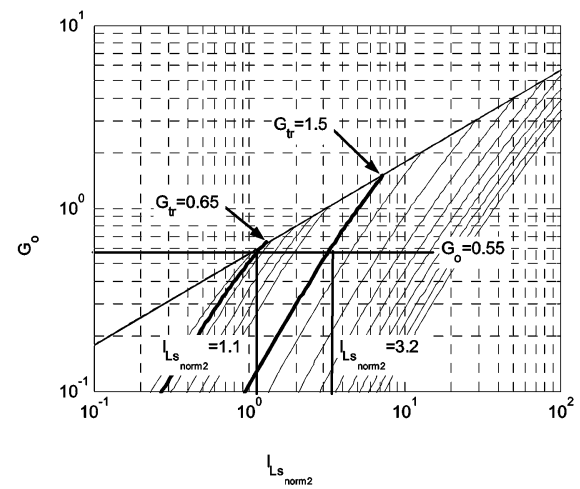

(e)

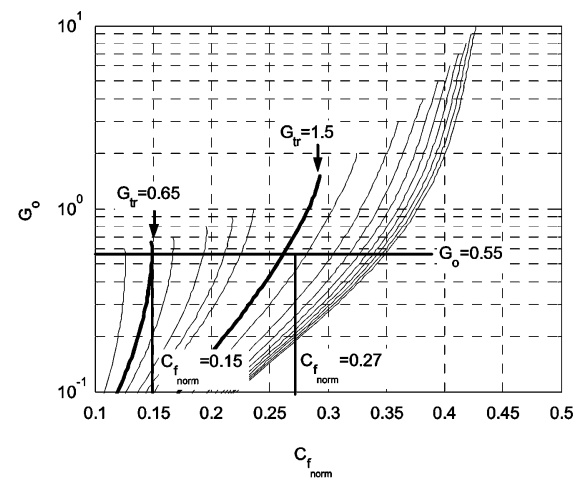

(h)

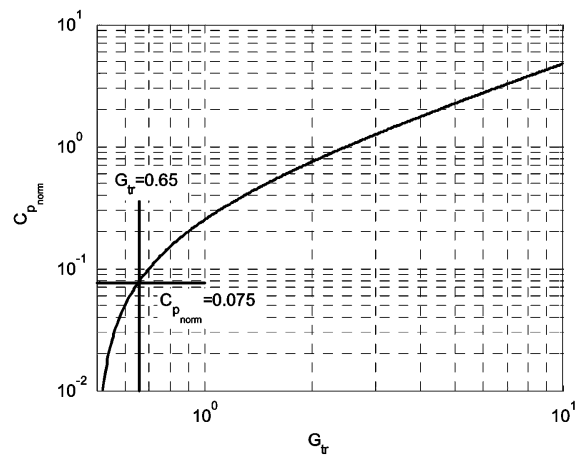

(c)

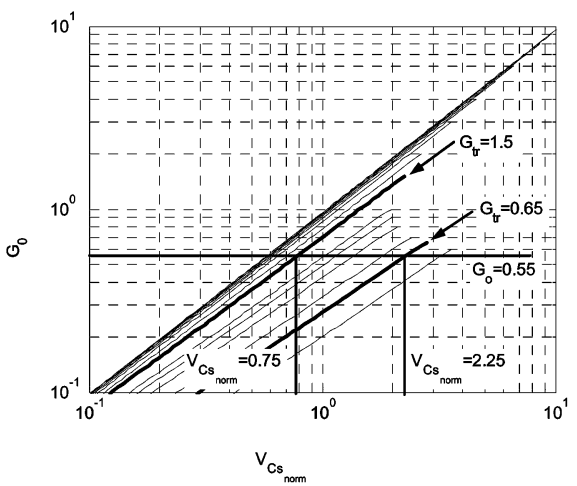

(f)

Fig. 16. Design curves for $A=1$ : (a) magnitude response, (b) phase response, (c) required parallel capacitor, (d) required inductor, (e) resonant inductor peak current, (f) peak series capacitor voltage stress, (g) resonant tank gain calculation curve, (h) required filter capacitor. (c) $C_{p}=C_{p_{\text {norm }}}\left(1 / N^{2} R_{L} f_{r}\right)=\left(2 G_{\text {tr }}-\right.$ $\left.1 / 4 N^{2} R_{L} f_{r}\right)(\mathrm{d}) L_{s}=L_{s_{\mathrm{norm}}}\left(N^{2} R_{L} / f_{r}\right) \approx\left(N^{2} R_{L} / f_{r}\left(9.755 G_{\mathrm{tr}}-3.061\right)\right), G_{\mathrm{tr}}>1$ (e) $\hat{I}_{L s}=I_{L s_{\mathrm{norm} 2}}\left(V_{i} / N^{2} R_{L}\right)(\mathrm{f}) V_{C s}=V_{C s_{\mathrm{norm}}} V_{i}(\mathrm{~h}) C_{f}=$ $C_{f_{\text {norm }}}\left(V_{o} / R_{L} f_{r} V_{\mathrm{pp}}\right)$.

Step 1) Calculate required effective load $\left(R_{L}^{\prime}\right)$ and tank gain $\left(G_{o}^{\prime}\right)$-MUR1560 diodes are to be used for the fullbridge rectifier. When the converter is operated at the peak output power, $I_{f}=1.2 \mathrm{~A}$, giving, from datasheets $V_{d}=0.9 \mathrm{~V}$. For use with the design curves, from (51) and (52), the minimum load and tank gain are therefore $R_{L}^{\prime}=16.5 \Omega, G_{o}^{\prime}=0.55$, respectively.

Step 2) Select a transformer turns ratio-From (6) it was demonstrated that $G_{\mathrm{tr}}>1 / 2$. It can therefore be shown that providing $N \geq 0.5 V_{i} /\left(V_{o_{\max }}+2 V_{d}\right)$ a converter design exists meeting the specifications. To ensure that the converter operates above resonance at the desired output voltage, the converter shall be designed for a maximum output voltage (at resonance) of $22 \mathrm{~V}(\approx 20 \%$ larger than required). A minimum value of $N$ is therefore 0.75 . However, for cost and volume reduction, the converter is not considered to include a transformer, and therefore, for calculation purposes, it is assumed that $N=1$.

Step 3) Calculate the required $G_{\mathrm{tr}}$-From Fig. 16(b), the first curve that meets the specification corresponds to $G_{\mathrm{tr}}=0.65$, as highlighted $\left(0.65^{*} V_{i} / N-2 \mathrm{Vd}=\right.$ $21.6 \mathrm{~V})$, and the phase shift is approximately $30^{\circ}$ above resonance. Also, from Fig. 15(a) $f_{m}=1.07$. To ensure that the nominal gain occurs at the specified switching frequency, the resonant frequency is chosen to be $f_{r}=93.5 \mathrm{kHz}$. 
Step 4) Calculate ideal resonant tank components-From Fig. 16(c)-(d), the normalized parallel capacitor and series inductor are found to be 0.075 and $2.5^{-1}$, respectively, corresponding to actual values of $48.6 \mathrm{nF}$ and $70.6 \mu \mathrm{H}$.

Step 5) Utilize preferred components-To obtain preferred values, while maintaining $G_{\mathrm{tr}}$, component values should be chosen to maintain $L_{s} / C_{p}$ and $A$ constant (see (34)). If $C_{p}=C_{s}=47 \mathrm{nF}$ and $L_{s}=68 \mu \mathrm{H}$, the requirement is satisfied. The new resonant frequency must now be calculated. From (35), since $A$ and $G_{\mathrm{tr}}$ have remained constant, the resonant frequency will increase by a factor of 1.036 to $96.9 \mathrm{kHz}$. The switching frequency will therefore have to be also increased to $103.6 \mathrm{kHz}$.

Step 6) Filter capacitor selection-Finally, from Fig. 16(h) the normalized filter capacitor required to limit the output voltage ripple to $10 \%$ is 0.15 , corresponding to $10 \mu \mathrm{F}$.

Step 7) Electrical stresses at $R_{L}=R_{o}$-From Fig. 16(e),(f) an indication of electrical component stresses can be found. The normalized peak inductor current and peak series capacitor voltage, are 1.1 and 2.25 , respectively, corresponding to actual values of $2.4 \mathrm{~A}$ and $81 \mathrm{~V}$.

Step 8) Upper switching frequency estimate-From Fig. 16(g) an estimate of the switching frequency to maintain the output voltage for the minimum load $(90 \Omega)$ can be obtained. An increase from $15 \Omega$ to $90 \Omega$ gives bounds for $R_{x}$ i.e., $R_{x}=1$ to $R_{x}=6$. When $G_{\mathrm{tr}}=0.65$, Fig. $16(\mathrm{~g})$ gives a $F_{3}\left(G_{t r}\right)$ value of 0.45 . Since the load increases by a factor of 6 the resonant tank gain needs to be found which gives a $F_{3}\left(G_{t r}\right)$ value of 2.7, which corresponds to $G_{\mathrm{tr}}=1.6$. From (45) the "new" resonant frequency is a factor of 1.22 higher than that at nominal load, giving $118.2 \mathrm{kHz}$. Now, from Fig. 16(a), extrapolating from the highlighted $G_{\mathrm{tr}}=1.5$ curve, for $G_{\mathrm{tr}}=1.6, f_{m} \approx 1.22$ and from Fig. 16(b) the phase shift is approximately $73^{\circ}$. The "new" switching frequency is therefore $144.2 \mathrm{kHz}$. Of note in this case is that if the "new" switching frequency exceeds the specification, from Fig. 17, given $A=1$, the value of $G_{\text {tro }}$ needs to be increased for regulation. In this case it is prudent to incorporate a transformer to increase the effective turns ratio. Additionally, if it is desired to vary $A$ while maintaining $G_{\text {tro }}$ and $N$, from Fig. 17 a larger $A$ achieves the same effect. However a new set of design curves for the alternative $A$ is required.

Step 9) Component stresses at maximum load resistanceThe normalized peak current stress is 3.2 and normalized peak series capacitor voltage is 0.75 , corresponding to $1.2 \mathrm{~A}$ and $27 \mathrm{~V}$, respectively. Note that the $90 \Omega$ load is scaled to compensate for the rectifier diode losses using (52) to give $99 \Omega$. From Fig. 16(h) the normalized filter capacitance is now approximately 0.27 , giving a peak-to-peak output voltage ripple of $42 \mathrm{mV}$.

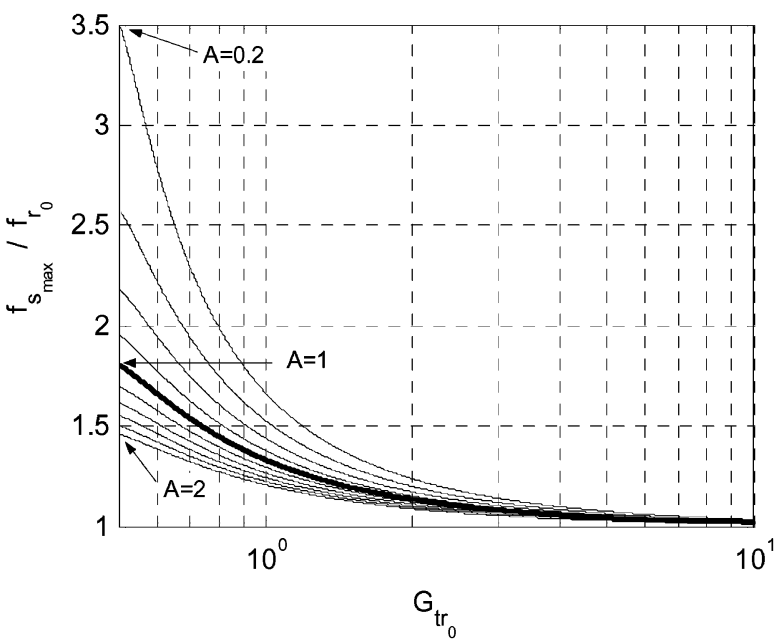

Fig. 17. Maximum bandwidth versus $G_{\text {tro }}$ for various $A$.

TABLE III

CONVERTER SPECIFICATIONS

\begin{tabular}{|c|c|c|}
\hline Input voltage & $V_{i}$ & $36 \mathrm{~V}$ \\
\hline Output voltage & $V_{o}$ & $18 \mathrm{~V}$ \\
\hline Load range & $R_{L}$ & $15 \rightarrow 90 \Omega,(21 \mathrm{~W} \rightarrow 4 \mathrm{~W})$ \\
\hline Switching frequency range & $f_{s_{\min }} \rightarrow f_{s_{\max }}$ & $100 \mathrm{kHz} \rightarrow 150 \mathrm{kHz}$ \\
\hline Parallel to series capacitor ratio & $A$ & 1 \\
\hline Ripple voltage & $V_{p p}$ & $0.18 \mathrm{~V}$ \\
\hline
\end{tabular}

TABLE IV

EXPERIMENTAL MEASUREMENTS VERSUS THEORETICAL ESTIMATES

\begin{tabular}{l|c|c|c|c}
\cline { 2 - 5 } & \multicolumn{2}{|c|}{$15 \Omega$ Load } & \multicolumn{2}{c}{$90 \Omega$ Load } \\
\cline { 2 - 5 } & Theoretical & Experimental & Theoretical & Experimental \\
\hline$f_{s}(\mathrm{kHz})$ & 103.6 & 101.3 & 144.2 & 143 \\
\hline$\hat{I}_{L S}(\mathrm{~A})$ & 2.4 & 2.3 & 1.2 & 1.2 \\
\hline$\hat{V}_{c s}(\mathrm{~V})$ & 81 & 85 & 27 & 27 \\
\hline$\theta\left(^{\circ}\right)$ & 30 & 27 & 73 & 77 \\
\hline$V_{p p}(\mathrm{~V})$ & 0.18 & 0.25 & 0.04 & 0.05 \\
\hline
\end{tabular}

To verify the design procedure experimentally, a converter was built with the calculated preferred component values. Results from experiments are summarized in Table IV, from where it can be seen that a high degree of correlation exists between the experimental and theoretical performance characteristics obtained from the analysis results and design procedure.

\section{Using HARMONICS TO TRANSFER POWER}

The possibility to transfer real power on harmonics, at the resonant frequency, has been discussed in Section II-B to provide a mechanism for reducing peak current stresses. Here, similar curves to those presented in Section VII (see Fig. 13, the Traditional design), are given for an $A$ and $G_{\mathrm{tr}}$ that produce a current reduction at resonance (the Harmonic power transfer design). Regulation performance can then be determined. Component stress and regulation curves have been generated for a converter 


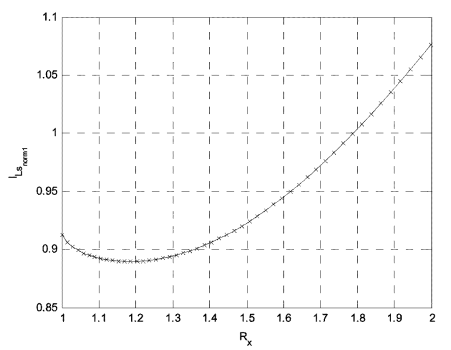

(a)

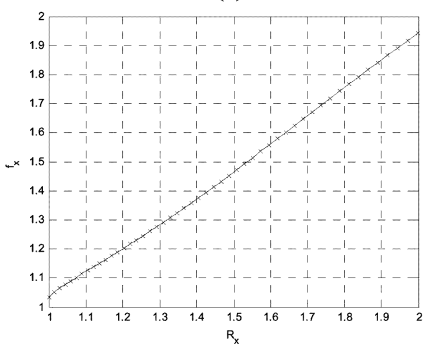

(c)

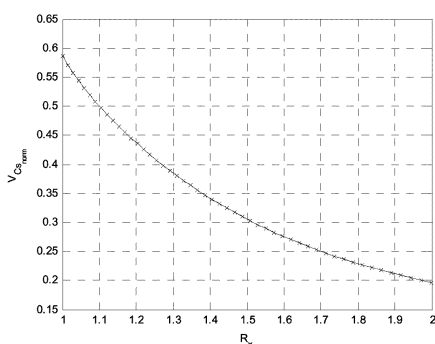

(b)

(d)

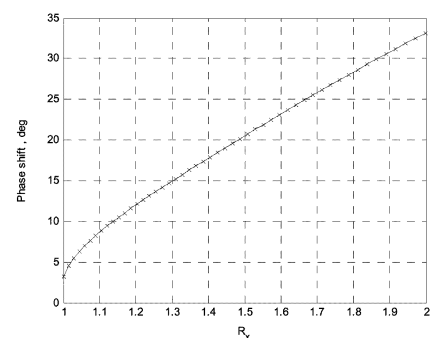

Fig. 18. (a), (b), (c), and (d) provide the regulation performance curves for a converter with $A=0.1$ and $G_{\mathrm{tr}}=0.55$ at nominal load. (a) Peak resonant current normalized to a sinusoid at resonance $\left(\hat{I}_{L s}=I_{L s_{\text {norm } 1}}\left(V_{i} / N^{2} R_{o}\right)\left(\pi G_{o}^{2} / R_{x}\right)\right)$. (b) Peak series capacitor voltage stress $\left(\hat{V}_{C s}=V_{C s_{\text {norm }}} V_{i}\right)$. (c) Regulation switching frequency normalized to nominal load $\left(f_{s}=f_{x} f_{\text {ro }}\right)$. (d) Phase shift required for output regulation.

with $A=0.1$ and $G_{\text {tr }}=0.55$, (the Harmonic power transfer design), in Fig. 18.

Of note, from Fig. 18(a), is that a reduction in the normalized peak current occurs as $R_{L}$ is increased indicating that the tank current wave-shape is becoming increasingly "square" as load resistance is increased. Comparing the peak normalized series-inductor current in Fig. 13(a) with that in Fig. 18(a), it can be seen that the Harmonic power transfer design has $\approx 9 \%$ lower normalized peak current at nominal load $\left(R_{x}=1\right), \approx 22 \%$ lower $I_{L s n o r m 1}$ at $R_{x}=1.2$ and $\approx 37 \%$ lower $I_{L s n o r m 1}$ at $R_{x}=2$ with respect to the Traditional design. This implies that the Harmonic power transfer design will have improved part-load efficiency (over the load range $R_{x}=1 \rightarrow 2$ ). An alternative strategy would be to design a converter such that the nominal load occurs at the minimum normalized current, thereby reducing stresses and further improving efficiency. Furthermore, by comparing the series capacitor stresses, from Figs. 13(a) and 18(a), it can be seen that the voltage is approximately $70 \%$ lower at nominal load in theHarmonic power transfer converter design. From Fig. 18(a) it can be seen that at $R_{x}=2$, the normalized current $>1$ hence the harmonics are no longer able to compensate for the reduced power factor. The stress reduction at this load is therefore mainly due to the much closer phase angle between switching voltage and series inductor current $\left(23^{\circ}\right)$ compared with theTraditional design $\left(53^{\circ}\right)$, see Figs. 18(d) and 13(e), respectively.

To verify the normalized stress reduction using an ideal SPICE model, the two designs were generated around the input/output and minimum load specifications provided in Table III. For theTraditional design where $A=1$ and $G_{\mathrm{tr}}=$ 1 , the ideal components required are $N=2, L_{s}=91.6 \mu \mathrm{H}$, $C_{s}=C_{p}=42.4 \mathrm{nF}$ and the Harmonic power transfer design
TABLE V

SPICE Simulation STRESS COMPARISON BETWEEN HARMONIC POWER TRANSFER AND TRADITIONAL CONVERTER DESIGN

\begin{tabular}{|c|c|c|c|c|}
\hline & $R_{L}(\Omega)$ & $f_{\mathrm{s}}(\mathrm{kHz})$ & $\hat{I}_{L s}(\mathrm{~A})$ & $\hat{V}_{C s}(\mathrm{~V})$ \\
\hline$A=1, G_{t r}=1$ & 15 & 100 & 1.91 & 71.4 \\
\hline 'Old' Design & 30 & 117.4 & 1.60 & 51.1 \\
\hline$A=0.1, G_{t r}=0.55$ & 15 & 100 & 1.72 & 21.1 \\
\hline 'New' Design & 30 & 188.1 & 1.01 & 7.1 \\
\hline
\end{tabular}

TABLE VI

HARMONIC CONTENT OF HARMONIC POWER TRANSFER CONVERTER DESIGN AT VARIOUS LOADS

\begin{tabular}{|c|c|c|c|c|c|c|}
\hline Harmonic & $\mathrm{R}_{\mathrm{L}(\Omega)}$ & $\hat{I}_{L s}(\mathrm{~A})$ & $\hat{V}_{s}(\mathrm{~V})$ & Phase $\left({ }^{\circ}\right)$ & Real Power $(\mathrm{W})$ & $\%$ Power \\
\hline \multirow{2}{*}{ Fundamental } & 15 & 1.83 & 22.9 & 6 & 20.9 & $96.8 \%$ \\
\cline { 2 - 7 } & 22.5 & 1.54 & 22.9 & 8.4 & 17.4 & $96.7 \%$ \\
\hline \multirow{2}{*}{$3^{\text {s. }}$} & 15 & 0.17 & 7.64 & 25.4 & 0.59 & $2.7 \%$ \\
\cline { 2 - 7 } & 22.5 & 0.22 & 7.64 & 49.9 & 0.55 & $3.1 \%$ \\
\hline \multirow{2}{*}{5 th } & 15 & 0.086 & 4.58 & -50 & 0.13 & $0.6 \%$ \\
\cline { 2 - 7 } & 22.5 & 0.093 & 4.58 & 84.6 & 0.02 & $0.1 \%$ \\
\hline
\end{tabular}

where $A=0.1$ and $G_{\mathrm{tr}}=0.55$ the components required are $N=1.1, L_{s}=25.5 \mu \mathrm{H}, C_{s}=142.2 \mathrm{nF}$ and $C_{p}=14.22 \mathrm{nF}$.

It can be seen that the data in Table $\mathrm{V}$ closely matches that predicted from the normalized analysis.

To show the ability of harmonics to carry power, Table VI provides the harmonic content when the output is regulated at $R_{x}=1$ and $R_{x}=1.2$ for the "new" converter design, along with the proportion of power transferred to the load. In particular, it can be seen that for the $22.5 \Omega$ case, the third harmonic transfers approximately $3 \%$ of the total output power, although, importantly, this corresponds to an effective reduction in the peak electrical stresses incurred due to $\hat{I}_{L s}$, of $\approx 10 \%$.

A consequence of reducing the electrical stresses, however, is that the switching bandwidth required to provide regulation over the whole load range, increases. Specifically, theTraditional converter required only $\approx 20 \%$ increase in resonant frequency to maintain the gain, whereas the Harmonic power transfer converter design requires $\approx 90 \%$ increase in frequency over the same range. This again indicates that utilizing harmonics to carry power is more suited to applications were the load is expected to have limited variability.

\section{CONCLUSION}

Normalized behavior of the LCC voltage-output converter characteristics, in terms of tank gain at resonance and the parallel capacitor to series capacitor ratio, has been presented. Design curves are derived that consider component value selection and related performance, including electrical stresses, switching frequency, phase shift, and tank gain at resonance, as the load is increased proportionally from the nominal load and the converter gain regulated at its nominal level. The design curves demonstrate that the entire behavior of the converter can be depicted graphically. Key features of paper are:

- an accurate relationship between the tank gain at resonance and the resonant frequency as the load is varied; 
- an alternative $Q$-factor expression based on energy stored in the resonant tank, and power dissipated in the load;

- examples demonstrating how to simply alter overall converter gain and switching frequency while maintaining the tank gain at resonance.

- expressions giving the maximum theoretical switching bandwidth to regulate the converter gain from nominal load to open circuit;

- an investigation into how the choice of nominal resonant tank gain and capacitor ratio affects part load efficiency.

The resulting methodology clearly defines the boundary between heavy and light loading conditions, in terms of normalized switching frequency or normalized load, and is ideally suited for use in a graphical user interface where design curves could form a basis for a constrained converter optimization. The underlying analysis has also identified operating regions where harmonics can transfer real power, thereby reducing peak current and component stresses. A design example is provided and results compared with experimental measurements on a prototype converter that demonstrate a high degree of correlation between theoretical and experimental performance characteristics. The design example also demonstrates how to select preferred component values and how the effects of rectifier voltage drop can be included in the design procedure.

Finally, it should be noted that although the analysis equations are derived for a bipolar input voltage, ultimately, the results are equally applicable to converters considered to have a unipolar input.

\section{APPENDIX}

\section{A. HEAVY LOADING}

Equations (38), (39) can be solved simultaneously to give

$$
\begin{aligned}
Q_{M 1} & =\frac{V_{i} G_{o}\left(2 G_{o}-f_{m}\left(2 G_{\mathrm{tr}}-1\right)-1\right)}{4 f_{r} f_{m} N^{2} R_{L}} \\
Q_{M 3} & =\frac{V_{i} G_{o}\left(2 G_{o}-f_{m}\left(2 G_{\mathrm{tr}}-1\right)+1\right)}{4 f_{r} f_{m} N^{2} R_{L}} .
\end{aligned}
$$

Since all the tank current flows through $C_{s}$

$$
\begin{aligned}
V_{C s_{M 4}}\left(t_{M 4}\right)=-V_{C s_{M 2}}(0) & =V_{C s_{M 2}}(0) \\
& +\frac{1}{C_{s}}\left(-Q_{\mathrm{M} 1}+Q_{\mathrm{M} 2}+Q_{\mathrm{M} 3}\right) .
\end{aligned}
$$

An expression for $V_{C s}$ at the start of $\mathrm{M} 2$ can be obtained from (6), (7), (39), and (A1)-(A3) as follows:

$$
V_{C s_{M 2}}(0)=\frac{-A V_{i} G_{o}\left(1+\left(2 G_{\mathrm{tr}}-1\right) f_{m}\right)}{\left(2 G_{\mathrm{tr}}-1\right) f_{m}} .
$$

An expression for $I_{L s}$ during M1 is determined by noting that $C_{e}=C_{s}$, since the $V_{C s}$ is clamped, see Fig. 9(a)

$$
\begin{aligned}
i_{L s_{M 1}}(t) & =\hat{I}_{M 1} \sin \left(\sqrt{\frac{1}{L_{s} C_{e}}}\left(t-t_{M 1}\right)\right) \\
& =\hat{I}_{M 1} \sin \left(\frac{A}{L_{s} C_{P}}\left(t-t_{M 1}\right)\right) .
\end{aligned}
$$

At the end of M1, $V_{L s}$ is given by

$$
V_{L s_{M 2}}(0)=L_{s} \frac{d i_{L s_{M 1}}\left(t_{M 1}\right)}{d t}=\frac{V_{i}}{2}+N V_{o}-V_{C s_{M 2}}(0) .
$$

Substituting (36), (A4) into (A6) and solving for the derivative gives

$$
\begin{aligned}
& \frac{d i_{L_{s_{M}}}(0)}{d t}=\frac{V_{i}}{2 L_{s}}+\frac{V_{i} G_{o}}{L_{s}} \\
& \quad+\frac{A V_{i} G_{o}\left(1+\left(2 G_{\mathrm{tr}}-1\right) f_{m}\right)}{L_{s}\left(2 G_{\mathrm{tr}}-1\right) f_{m}}
\end{aligned}
$$

Equating the derivative in (A7) with the time derivative in (A5), at $t=t_{\mathrm{M} 1}$, and solving for $\hat{I}_{M 1}$ gives

$$
\hat{I}_{M 1}=\frac{V_{i}\left(f_{m}\left(2 G_{\mathrm{tr}}-1\right)\left(1+2(A+1) G_{o}\right)+2 A G_{o}\right)}{4 f_{m} N \sqrt{R_{L} A L_{s} f_{r}\left(2 G_{\mathrm{tr}}-1\right)}} .
$$

It is now possible to calculate the time spent in M1 $\left(t_{M 1}\right)$. Substituting (6), (A1), (A5), (A8) into (1) and solving for $t_{M 1}$ gives

$$
\begin{aligned}
t_{M 1}= & \frac{1}{2 N} \sqrt{\frac{L_{s}\left(2 G_{\mathrm{tr}}-1\right)}{R_{L} A f_{r}}} \cos ^{-1} \\
& \left(\frac{f_{m}\left(2 G_{\mathrm{tr}}-1\right)\left(1+2 G_{o}\right)+4 \mathrm{AG}_{o}^{2}}{f_{m}\left(2 G_{\mathrm{tr}}-1\right)\left(1+2(A+1) G_{o}\right)+2 A G_{o}}\right) .
\end{aligned}
$$

Substituting (A8) and (A9) into (A5) gives $I_{L s}$ during M1.

The tank current during M2 is now derived by noting that during $\mathrm{M} 2 C_{e}$ is the series combination of $C_{s}$ and $C_{p}$, see Fig. 9(b). The tank current then takes the form

$$
\begin{aligned}
i_{L s_{M 2}}(t) & =\hat{I}_{M 2} \sin \left(t \sqrt{\frac{1}{L_{s} C_{e}}}\right) \\
& =\hat{I}_{M 2} \sin \left(t \sqrt{\frac{A+1}{L_{s} C_{P}}}\right) .
\end{aligned}
$$

Equating the time derivative of (A10) at $t=0$ to (A7), and solving for $\hat{I}_{M 2}$ provides

$$
\begin{aligned}
\hat{I}_{M 2} & =\frac{V_{i}\left(f_{m}\left(2 G_{\mathrm{tr}}-1\right)\left(1+2(A+1) G_{o}\right)+2 A G_{o}\right)}{4 f_{m} N \sqrt{R_{L}(A+1) L_{s} f_{r}\left(2 G_{\mathrm{tr}}-1\right)}} \\
& =\hat{I}_{M 1} \sqrt{\frac{A}{A+1}}
\end{aligned}
$$

and, substituting (6), (39), (A10), and (A11) into (1) and solving for $t_{M 2}$ gives

$$
\begin{aligned}
t_{M 2}= & \frac{1}{2 N} \sqrt{\frac{L_{s}\left(2 G_{\mathrm{tr}}-1\right)}{R_{L}(A+1) f_{r}}}\left(\pi-\cos ^{-1}\right. \\
& \left.\left(\frac{f_{m}\left(2 G_{\mathrm{tr}}-1\right)\left(-1+2(1+A) G_{o}\right)-2 A G_{o}}{f_{m}\left(2 G_{\mathrm{tr}}-1\right)\left(1+2(A+1) G_{o}\right)+2 A G_{o}}\right)\right) .
\end{aligned}
$$


$I_{L s}$ at the end of M2 is obtained by substituting (6), (A11), (A12) into (A10) (i.e., $t=t_{M 2}$ )

$$
i_{L s_{M 3}}(0)=\frac{V_{i}}{N} \sqrt{\frac{G_{o}\left(\left(2 G_{\mathrm{tr}}-1\right) f_{m}+2 A G_{o}\right)}{2 R_{L} f_{r} f_{m} L_{s}}} .
$$

The derivative of $I_{L s}$ at the end of M2 is obtained by substituting (6), (A11), (A12) into the time derivative of (A10) (i.e., $\left.t=t_{M 2}\right)$

$$
\begin{aligned}
& \frac{d i_{L s_{M 3}}(0)}{d t} \\
& =\frac{V_{i}\left(f_{m}\left(2 G_{\mathrm{tr}}-1\right)\left(1-2(A+1) G_{o}\right)+2 A G_{o}\right)}{2 L_{s}\left(2 G_{\mathrm{tr}}-1\right) f_{m}} .
\end{aligned}
$$

During M3, $C_{e}=C_{s}$ see Fig. 9(c), and, by application of Laplace transforms, the tank current during M3 will have the following form:

$$
\begin{aligned}
i_{L s_{M 3}}(t) & =\hat{I}_{M 3} \cos \left(t \sqrt{\frac{1}{L_{s} C_{e}}}+\phi\right) \\
& =\hat{I}_{M 3} \cos \left(t \sqrt{\frac{A}{L_{s} C_{P}}}+\phi\right)
\end{aligned}
$$

where

$$
\begin{aligned}
\hat{I}_{M 3} & =i_{L s_{\mathrm{M} 3}}(0) \sqrt{1+\frac{L_{s} C_{p}\left(\frac{d i_{L s_{M 3}}(0)}{d t}\right)^{2}}{A i_{L s_{M 3}}(0)^{2}}}, \\
\phi & =-\tan ^{-1}\left(\frac{1}{i_{L s_{M 3}}(0)} \sqrt{\frac{L_{s} C_{p}}{A}} \frac{d i_{L s_{M 3}}(0)}{d t}\right)
\end{aligned}
$$

where $i_{L s_{M 3}}(0),\left(d i_{L s_{M 3}}(0) / d t\right)$ are the initial conditions of the mode and have been calculated in (A13), (A14), respectively. Substituting (6), (A13), and (A14) into (A15) provides the expression for $I_{L s}$ during M3.

The magnitude of $i_{L_{M 3}}(t)$ is (A16), shown at the bottom of the page.

Substituting (6), (A2), and (A13)-(A16) into (1) and solving for $t_{\mathrm{M} 3}$ finally provides the time spent in M3 as (A17), shown at the bottom of the page.

\section{APPENDIX}

\section{B. LIGHT LOADING}

Equations (40), (41) can be solved simultaneously to give the following:

$$
\begin{aligned}
Q_{M 1} & =\frac{V_{i} G_{o}\left(2 G_{o}-f_{m}\left(2 G_{\mathrm{tr}}-1\right)+1\right)}{4 f_{r} f_{m} N^{2} R_{L}} \\
Q_{M 2} & =\frac{-V_{i} G_{o}}{2 f_{m} f_{r} N^{2} R_{L}} \\
Q_{M 3} & =\frac{V_{i} G_{o}\left(2 G_{o}+f_{m}\left(2 G_{\mathrm{tr}}-1\right)+1\right)}{4 f_{r} f_{m} N^{2} R_{L}} .
\end{aligned}
$$

Since all the tank current flows though $C_{\mathrm{s}}$, the change in $V_{C s}$ during the positive half-cycle of the tank current is

$$
\begin{aligned}
V_{C s_{M 5}}\left(t_{M 5}\right)=-V_{C s_{3}}(0) & =V_{C s_{M 3}}(0) \\
+ & +\frac{1}{C_{s}}\left(-Q_{M 1}-Q_{M 2}+Q_{M 3}\right) .
\end{aligned}
$$

$V_{C s}$ at the start of M3 follows from (6), (7), (A18)-(A20), (A21)

$$
V_{C s_{M 3}}(0)=\frac{-A V_{i} G_{o}\left(1+\left(2 G_{\mathrm{tr}}-1\right) f_{m}\right)}{\left(2 G_{\mathrm{tr}}-1\right) f_{m}} .
$$

Notably, this is the same for $V_{C s}$ at the start of M2 for operation under heavy loading (A4).

During M2, $C_{e}=C_{s}$, see Fig. 11(b), and

$$
i_{L s_{M 2}}(t)=\hat{I}_{M 2} \sin \left(\sqrt{\frac{A}{L_{s} C_{P}}}\left(t-t_{M 2}\right)\right) \text {. }
$$

At the end of $\mathrm{M} 2, V_{L s}$ is given by

$$
\begin{aligned}
V_{L s_{M 2}}\left(t_{M 2}\right) & =L_{s} \frac{d i_{L s_{M 2}}\left(t_{M 2}\right)}{d t} \\
& =\frac{V_{i}}{2}+N V_{o}-V_{C s_{M 3}}(0) .
\end{aligned}
$$

Substituting (36), (A22)into (A24) and solving for the derivative gives

$$
\begin{aligned}
& \frac{d i_{L s_{M 2}}\left(t_{M 2}\right)}{d t} \\
& =\frac{V_{i}}{2 L_{s}}+\frac{V_{i} G_{O}}{L_{s}}+\frac{A V_{i} G_{O}\left(1+\left(2 G_{\mathrm{tr}}-1\right) f_{m}\right)}{L_{s}\left(2 G_{\mathrm{tr}}-1\right) f_{m}} .
\end{aligned}
$$

(A25)

$$
\hat{I}_{M 3}=\frac{V_{i}\left(\sqrt{f_{m}^{2}\left(2 G_{\mathrm{tr}}-1\right)^{2}\left(4(A+1)^{2} G_{o}^{2}+4(A-1) G_{o}+1\right)+f_{m}\left(2 G_{\mathrm{tr}}-1\right) 4 G_{o} A\left(2(A-1) G_{o}+1\right)+4 A^{2} G_{o}^{2}}\right)}{4 f_{m} N \sqrt{R_{L} A L_{s} f_{r}\left(2 G_{\mathrm{tr}}-1\right)}}
$$

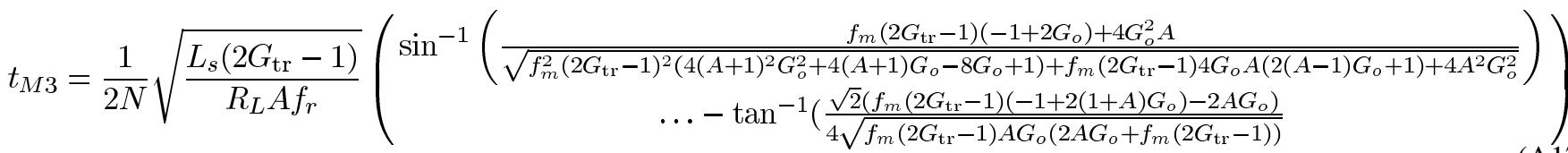


Equating the derivative of (A23) at $t=t_{M 2}$ with (A25) and solving for $\hat{I}_{M 2}$ provides

$$
\hat{I}_{M 2}=\frac{V_{i}\left(f_{m}\left(2 G_{\mathrm{tr}}-1\right)\left(1+2(A+1) G_{o}\right)+2 A G_{o}\right)}{4 f_{m} N \sqrt{R_{L} A L_{s} f_{r}\left(2 G_{\mathrm{tr}}-1\right)}} .
$$

Again, this is the same result as for $\hat{I}_{\mathrm{M} 1}$ during heavily loaded operation.

The time spent in M2 $\left(t_{M 2}\right)$ can be obtained by substituting (6), (A19), (A23), (A26) into (1) and solving for $t_{M 2}$

$$
\begin{aligned}
t_{M 2}= & \frac{1}{2 N} \sqrt{\frac{L_{s}\left(2 G_{\mathrm{tr}}-1\right)}{R_{L} A f_{r}}} \cos ^{-1} \\
& \left(\frac{f_{m}\left(2 G_{\mathrm{tr}}-1\right)\left(1+2(A+1) G_{o}\right)-2 A G_{o}}{f_{m}\left(2 G_{\mathrm{tr}}-1\right)\left(1+2(A+1) G_{o}\right)+2 A G_{o}}\right) .
\end{aligned}
$$

Substituting (A26), (A27) into (A23) also gives $I_{L s}$ during $\mathrm{M} 2$.

$I_{L s}$ at the start of M2 is now be calculated by substituting (6), (A26), (A27) into (A23) and evaluating at $t=0$, giving

$$
i_{L s_{M 2}}(0)=-\frac{V_{i}}{N} \sqrt{\frac{G_{o}\left(2(A+1) G_{o}+1\right)}{2 R_{L} f_{r} f_{m} L_{s}}}=i_{L_{\mathrm{M} 1}}\left(t_{\mathrm{M} 1}\right) .
$$

The derivative at the start of $\mathrm{M} 2$ is similarly found by substituting (6), (A26), (A27) into the derivative of (A23) and evaluating at $t=0$

$$
\begin{aligned}
& \frac{d i_{L s_{M 2}}(0)}{d t} \\
& =\frac{V_{i}\left(f_{m}\left(2 G_{\mathrm{tr}}-1\right)\left(2 G_{o}(1+A)+1\right)-2 G_{O} A\right)}{2 f_{m}\left(2 G_{\mathrm{tr}}-1\right) L_{s}} \\
& =\frac{d i_{L s_{M 1}}\left(t_{M 1}\right)}{d t} .
\end{aligned}
$$

$I_{\mathrm{Ls}}$ during M3 is obtained by noting that $C_{e}$ is the series combination of $C_{\mathrm{s}}$ and $C_{p}$ [see Figs. 11(c) and (9)], and, therefore

$$
i_{L s_{M 3}}(t)=\hat{I}_{M 3} \sin \left(t \sqrt{\frac{1}{L_{s} C_{e}}}\right)=\hat{I}_{M 3} \sin \left(t \sqrt{\frac{A+1}{L_{s} C_{P}}}\right) .
$$

Equating the derivative of $I_{L s}$ at the start of M3 with that at the end of M2, gives $\hat{I}_{M 3}$. Consequently, equating the derivative of (A30) at $t=0$ with (A25) (the derivative of (A23) at $t=$ $t_{M 2}$ ), and solving for $\hat{I}_{M 3}$, ultimately provides

$$
\begin{aligned}
\hat{I}_{M 3} & =\frac{V_{i}\left(f_{m}\left(2 G_{\mathrm{tr}}-1\right)\left(1+2(A+1) G_{o}\right)+2 A G_{o}\right)}{4 f_{m} N \sqrt{R_{L}(A+1) L_{s} f_{r}\left(2 G_{\mathrm{tr}}-1\right)}} \\
& =\hat{I}_{M 2} \sqrt{\frac{A}{A+1}} .
\end{aligned}
$$

Finally, substituting (A31) into (A30) gives $I_{L s}$ during M3, and substituting (6), (A20), (A30), (A31) into (1) and solving for $t_{M 3}$ gives

$$
\begin{aligned}
t_{M 3}= & \frac{1}{2 N} \sqrt{\frac{L_{s}\left(2 G_{\mathrm{tr}}-1\right)}{R_{L}(A+1) f_{r}}} \\
& \times \cos ^{-1}\left(\frac{f_{m}\left(2 G_{\mathrm{tr}}-1\right)-2 G_{o}\left(1+2(A+1) G_{o}\right)}{f_{m}\left(2 G_{\mathrm{tr}}-1\right)\left(1+2(A+1) G_{o}\right)+2 A G_{o}}\right) .
\end{aligned}
$$

$I_{L s}$ at the end of M3 is obtained by substituting (6), (A31), (A32) (i.e., $t=t_{M 3}$ ) into (A30), as (A33), shown at the bottom of the page.

The derivative of $I_{L s}$ at the start of M3 is similarly found by substituting (6), (A31), (A32) (i.e., $t=t_{M 3}$ ) into the derivative of (71)

$$
\frac{d i_{L s_{M 3}}\left(t_{M 3}\right)}{d t}=\frac{V_{i}\left(f_{m}\left(2 G_{\mathrm{tr}}-1\right)-2 G_{o}\left(2(A+1) G_{o}+1\right)\right)}{2\left(2 G_{\mathrm{tr}}-1\right) f_{m} L_{s}} .
$$

Since the converter is assumed to be operating in steady-state, $I_{L s}$ at the start of M1 is of opposite polarity to that at the end of $\mathrm{M} 3$, i.e.

$$
i_{L s_{M 1}}(0)=-i_{L s_{M 3}}\left(t_{M 3}\right)
$$

and the derivative of $I_{L s}$ at the start of M1 is also related to the derivative of $I_{L s}$ at the end of M3, by

$$
\frac{d i_{L s_{M 1}}(0)}{d t}=-\left(\frac{d i_{L s_{M 3}}\left(t_{M 3}\right)}{d t}-\frac{V_{i}}{L_{s}}\right) \text {. }
$$

The tank current during M1 takes the following form:

$$
i_{L s_{M 1}}(t)=\hat{I}_{M 1} \cos \left(t \sqrt{\frac{A+1}{L_{s} C_{P}}}+\phi\right)
$$

where

$$
\begin{aligned}
\hat{I}_{M 1} & =i_{L s_{M 1}}(0) \sqrt{1+\frac{L_{s} C_{p}\left(\frac{d i_{L s_{M 1}}(0)}{d t}\right)^{2}}{(A+1) i_{L s_{M 1}}(0)^{2}}}, \\
\phi & =-\tan ^{-1}\left(\frac{1}{i_{L s_{M 1}}(0)} \sqrt{\frac{L_{s} C_{p}}{(A+1)}} \frac{d i_{L s_{M 1}}(0)}{d t}\right)
\end{aligned}
$$

with initial conditions $i_{L s_{M 1}}(0),\left(d i_{L s_{M 1}}(0) / d t\right)$ that have been calculated in (A35) and (A36), respectively. Consequently, substituting (6) and (A33)-(A36) into (A37) provides the tank current during the $\mathrm{M} 1, i_{L s_{M 1}}(t)$, and the amplitude is (A38), shown at the top of the next page.

$$
\begin{aligned}
& i_{L s_{M 3}}\left(t_{M 3}\right)=\frac{V_{i}}{2 N f_{m}} \\
& \sqrt{\frac{G_{o}\left((G o(A+1)+1) f_{m}^{2}\left(2 G_{\mathrm{tr}}-1\right)^{2}+\left(2 G_{o}(A+1)+1\right) f_{m}\left(2 G_{\mathrm{tr}}-1\right)-\left(2 G_{O}+1\right) G_{o}\left(2 G_{o}(A+1)-A+1\right)\right.}{R_{L} f_{r} L_{s}\left(2 G_{\mathrm{tr}}-1\right)}}
\end{aligned}
$$




$$
\hat{I}_{M 1}=\frac{-V_{i}\left(\sqrt{f_{m}^{2}\left(2 G_{\mathrm{tr}}-1\right)^{2}\left(2 G_{o}(1+A)+1\right)^{2}+f_{m}\left(2 G_{\mathrm{tr}}-1\right)\left(4 G_{o}(A+2)\left(2 G_{o}(1+A)+1\right)+4 G_{o}^{2} A\right.}\right)}{4 f_{m} N \sqrt{R_{L}(A+1) L_{s} f_{r}\left(2 G_{\mathrm{tr}}-1\right)}}
$$

$$
t_{M 1}=\frac{1}{2 N} \sqrt{\frac{L_{s}\left(2 G_{\mathrm{tr}}-1\right)}{R_{L}(A+1) f_{r}}}\left(\begin{array}{c}
\sin ^{-1}\left(\frac{f_{m}\left(2 G_{\mathrm{tr}}-1\right)\left(2 G_{o}(A+1)+1\right)-2 G_{o} A}{\sqrt{f_{m}^{2}\left(2 G_{\mathrm{tr}}-1\right)^{2}\left(2 G_{o}(A+1)+1\right)^{2}+f_{m}\left(2 G_{\mathrm{tr}}-1\right)\left(4 G_{o}(A+2)\left(2 G_{o}(1+A)+1\right)\right)+4 G_{o}^{2} A^{2}}}\right) \\
\ldots-\tan ^{-1}\left(\frac{f_{m}\left(2 G_{\mathrm{tr}}-1\right)+4 G_{o}^{2}(A+1)+2 G_{o}}{2 \sqrt{G_{o}\left(2 G_{o}+1+f_{m}\left(2 G_{\mathrm{tr}}-1\right)\right)(A+1)\left(\left((A+1) G_{o}+1\right) f_{m}\left(2 G_{\mathrm{tr}}-1\right)-G_{o}\left(2 G_{o}(A+1)+1-A\right)\right)}}\right)
\end{array}\right)
$$

Substituting (6), (A18), and (A33), (A34)-(A37) into (1) and solving for $t_{M 1}$ finally gives (A39), shown at the top of the page.

\section{REFERENCES}

[1] R. L. Steigerwald, Ed., "A comparison of half-bridge resonant converter topologies," IEEE Trans. Power Electron., vol. PE-3, no. 2, pp. 174-182, Apr. 1988.

[2] M. K. Kazimierczuk and D. Czarkowski, Resonant Power Converters. New York: Wiley, 1995, ch. 8 \& 17.

[3] A. K. S. Bhat, "Analysis and design of series-parallel resonant power supply," IEEE Trans. Aerosp. Electron. Syst., vol. 28, no. 1, pp. 249-258, Jan. 1992.

[4] M. K. Kazimierczuk, N. Thirunarayan, and S. Shan, "Analysis of series-parallel resonant converter," IEEE Trans. Aerosp. Electron. Syst., vol. 29, no. 1, pp. 88-99, Jan. 1993.

[5] M. P. Foster, H. I. Sewell, C. M. Bingham, D. A. Stone, and D. Howe, "Methodologies for the design of LCC voltage-output resonant converters," Proc. Inst. Elect. Eng., vol. 153, no. 4, pp. 559-567, Jul. 2006.

[6] A. J. Forsyth and S. V. Mollov, "Simple equivalent circuit for the series-loaded resonant converter with voltage boosting capacitor," Proc. Inst. Elect. Eng., vol. 145, no. 4, pp. 301-306, 1998.

[7] A. K. S. Bhat, "A generalized steady-state analysis of resonant converters using two-port model and fourier-series approach," IEEE Trans. Power Electron., vol. 13, no. 1, pp. 142-151, Jan. 1998.

[8] S.-C. Wong and A. D. Brown, "Analysis, modelling, and simulation of series-parallel resonant converter circuits," IEEE Trans. Power Electron., vol. 10, no. 5, pp. 605-614, Sep. 1995.

[9] M. P. Foster, H. I. Sewell, C. M. Bingham, and D. A. Stone, "State-variable modelling of LCC voltage output resonant converters," Electron. Lett., vol. 37, no. 17, pp. 1065-1066, 2001.

[10] A. K. S. Bhat, "Analysis and design of a series-parallel resonant converter with capacitive output filter," IEEE Trans. Ind. Appl., vol. 27, no. 3, pp. 523-530, May 1991.

[11] A. K. S. Bhat and S. B. Dewan, "Analysis and design of a high frequency resonant converter using LCC-type commutation," IEEE Trans. Power Electron., vol. PE-2, no. 4, pp. 291-301, Oct. 1987.

[12] I. Batarseh, R. Lie, C. Q. Lee, and A. K. Upadhyay, "Theoretical and experimental studies of the LCC-type parallel resonant converter," IEEE Trans. Power Electron., vol. PE-5, no. 2, pp. 140-150, Apr. 1990.

[13] R. P. Severns, "Topologies for three-element resonant converters," IEEE Trans. Power Electron., vol. 7, no. 1, pp. 89-98, Jan. 1992.

[14] I. Batarseh, "Resonant converter topologies with three and four energy storage elements," IEEE Trans. Power Electron., vol. 9, no. 1, pp. 64-73, Jan. 1994.

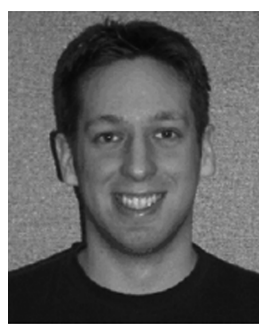

Adam J. Gilbert received the M.Eng. degree in electronic and electrical engineering from the University of Sheffield, Sheffield, U.K., in 2002 where he is currently pursuing the Ph.D. degree.

His research interests include the modelling and control of switching power converters with particular emphasis on high-efficiency resonant supplies.

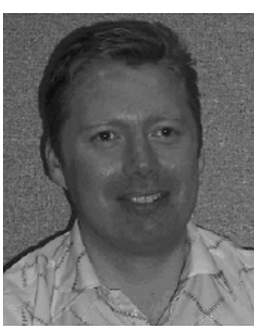

Christopher M. Bingham received the B.Eng. degree in electronic systems and control engineering from Sheffield City Polytechnic, Sheffield, U.K., in 1989, the M.Sc.(Eng.) degree in control systems engineering from the University of Sheffield, Sheffield, U.K., in 1990, and the Ph.D. degree in control systems to accommodate non-linear dynamic effects in aerospace flight-surface actuators from Cranfield University, Shrivenham, U.K., in 1994.

$\mathrm{He}$ remained at Cranfield University as a Post-Doctoral Researcher, until subsequently taking up a research position at the University of Sheffield. Since 1998, he has been a Lecturer in the Department of Electronic and Electrical Engineering, University of Sheffield. His current research interests include traction control/anti-lock braking systems for electric vehicles, electro-mechanical actuation of flight control surfaces, control of active magnetic bearings for high-speed machines, sensorless control of brushless machines, analysis and design of resonant converter systems, and the control of high-performance UAVs.

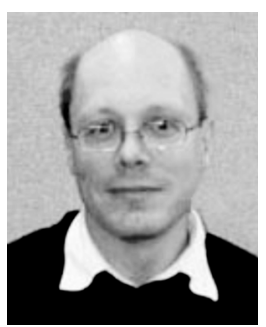

David A. Stone received the B.Eng. degree in electronic engineering from the University of Sheffield, Sheffield, U.K., in 1984 and the Ph.D. degree from Liverpool University, Liverpool, U.K., in 1989.

He then returned to the University of Sheffield as a member of academic staff specializing in power electronics and machine drive systems. His current research interests are in hybrid-electric vehicles, battery charging, EMC and novel lamp ballasts for low pressure fluorescent lamps.

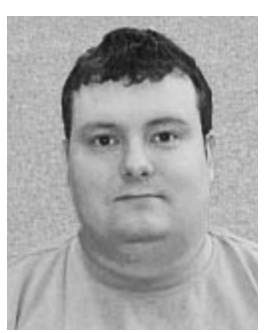

Martin P. Foster received the B.Eng. degree in electronic and electrical engineering, the M.Sc.(Eng.) degree in control systems, and the rapid analysis and design of resonant converters from the University of Sheffield, Sheffield, U.K., in 1998, 2000, and 2003, respectively.

His current research interests include the modeling and control of switching power converter with particular emphasis on high-efficiency resonant supplies, and novel control methods for autonomous aerospace vehicles. 